\title{
Multi-black holes from nilpotent Lie algebra orbits
}

\author{
Guillaume Bossard • Hermann Nicolai
}

Received: 16 June 2009 / Accepted: 15 July 2009 / Published online: 4 August 2009

(C) The Author(s) 2009. This article is published with open access at Springerlink.com

\begin{abstract}
For $\mathcal{N} \geq 2$ supergravities, BPS black hole solutions preserving four supersymmetries can be superposed linearly, leading to well defined solutions containing an arbitrary number of such BPS black holes at arbitrary positions. Being stationary, these solutions can be understood via associated non-linear sigma models over pseudo-Riemannian spaces coupled to Euclidean gravity in three spatial dimensions. As the main result of this paper, we show that whenever this pseudo-Riemannian space is an irreducible symmetric space $\mathfrak{G} / \mathfrak{H}^{*}$, the most general solutions of this type can be entirely characterised and derived from the nilpotent orbits of the associated Lie algebra $\mathfrak{g}$. This technique also permits the explicit computation of non-supersymmetric extremal solutions which cannot be obtained by truncation to $\mathcal{N}=2$ supergravity theories. For maximal supergravity, we not only recover the known BPS solutions depending on 32 independent harmonic functions, but in addition find a set of nonBPS solutions depending on 29 harmonic functions. While the BPS solutions can be understood within the appropriate $\mathcal{N}=2$ truncation of $\mathcal{N}=8$ supergravity, the general non-BPS solutions require the whole field content of the theory.
\end{abstract}

Keywords Supergravity $\cdot$ Black holes $\cdot$ Lie algebra

\section{Introduction}

Extremal static black holes with identical charges do not interact. As first pointed out by Papapetrou and Majumdar, for every spherically symmetric extremal black hole of

\footnotetext{
G. Bossard $(\varangle) \cdot$ H. Nicolai

AEI, Max-Planck-Institut für Gravitationsphysik, Am Mühlenberg 1, 14476 Potsdam, Germany

e-mail: bossard@aei.mpg.de

H. Nicolai

e-mail: Hermann.Nicolai@aei.mpg.de
} 
Maxwell-Einstein theory, one can arrive at associated multi-black hole solutions by means of linear 'superposition', that is, by substituting any harmonic function of the inverse radius in the potentials defining the stationary metric and the vector field [1,2]. Physically, the stability (or 'staticity') of these solutions is ensured by the 'no force' property, whereby the gravitational attraction between any two charged black holes is exactly balanced by their mutual electromagnetic repulsion. On the other hand, considering Maxwell-Einstein theory as the bosonic sector of $\mathcal{N}=2$ supergravity, one can understand this property mathematically from the fact that the corresponding solutions are BPS, as they preserve one-half (that is, four) of the supersymmetry charges. For general matter coupled $\mathcal{N}=2$ supergravity theories, there are multi-black hole solutions of BPS type for any black hole preserving four identical supersymmetry charges. Such solutions have been identified in a general way in [3], in the framework of the so-called attractor mechanism $[4,5]$.

However, the BPS multi-black hole solutions are not the most general solutions of this type. In this publication we characterise the general solutions of PapapetrouMajumdar type for all models for which the scalar fields coordinatise a symmetric space. More precisely, our discussion applies to all models containing gravity coupled to abelian vector fields and scalar fields parametrising a symmetric Riemannian space $\mathfrak{G}_{4} / \mathfrak{H}_{4}$ whose isometry group $\mathfrak{G}_{4}$ acts faithfully on the vector fields. For these models, the stationary equations of motion reduce to the ones of a non-linear sigma model defined over a pseudo-Riemannian symmetric space $\mathfrak{G} / \mathfrak{H}^{*}$ coupled to three-dimensional Euclidean gravity [6-8], with $\mathfrak{G}$ being a simple Lie group, and $\mathfrak{H}^{*}$ a non-compact real form of its maximal compact subgroup. The derivation of Papapetrou-Majumdar type solutions then reduces to certain algebraic conditions [9] (see Eqs. (3.2) and (3.5) of this paper). Using the formalism developed in [8], and especially the explicit relation between extremal solutions and nilpotent orbits of the three-dimensional duality group $\mathfrak{G}$, we are able to solve these algebraic equations in full generality. A crucial feature here is that, unlike supergravity duality groups in dimensions $D \geq 4$, the duality groups $\mathfrak{G}$ of the three-dimensional theories also incorporate the Ehlers symmetry $S L(2, \mathbb{R})$, and thus the gravitational degrees of freedom. The importance of Lie algebra nilpotent orbits in the physics of extremal black holes was first pointed out in [10]. The case of minimal supergravity in five dimensions has been studied in detail in this framework in [11].

In contrast to previous studies of BPS solutions, the analysis of [8] is solely based on exploiting properties of the $\mathfrak{G}$-Noether charge $\mathscr{C}$ and the associated BPS parameter $c$, which are defined in Eqs. (2.5) and (2.8) below. As shown in [8], the Noether charge satisfies a cubic or quintic characteristic equation, cf. (3.13) and (3.14), respectively. The latter determines the BPS parameter as a function of the physical charges of the four-dimensional theory, namely the complex gravitational charge $W=m+i n$ (with $m$ the mass and $n$ the Taub-NUT parameter) and the complex (dyonic) electromagnetic charges $Z_{A}=q_{A}+i p_{A}$, viz.

$$
c=c\left(W, Z_{A}\right)
$$

Solutions of Papapetrou-Majumdar type are superpositions of extremal black holes whose Noether charges satisfy the condition $c=0$. These solutions come in two 
categories. Those of the first category are $\frac{1}{2}$ BPS solutions of an appropriate truncation to a theory that constitutes the bosonic sector of some $\mathcal{N}=2$ supergravity theory coupled to $n_{V}$ vector multiplets. The $\frac{1}{2}$ BPS solutions of the latter theory define multiblack hole solutions depending on $2+2 n_{V}$ harmonic functions $\left(1+2 n_{V}\right.$ if one requires the solution to be asymptotically Minkowski). They define the most general solutions of Papapetrou-Majumdar type whenever the associated group $\mathfrak{H}^{*}$ is non-semi-simple, i.e. possesses a $U(1)$ factor. In this case, the BPS parameter can be written in the form

$$
c^{2}=\frac{\left(|W|^{2}-\left|z_{1}\right|^{2}\right)\left(|W|^{2}-\left|z_{2}\right|^{2}\right)}{|W|^{2}}
$$

where $z_{1}$ and $z_{2}$ generalise the central charges associated to the electromagnetic charges to theories which are not necessarily supersymmetric. It then follows that the extremality condition $c=0$ requires at least one of the charges to saturate the BPS bound $|z|=|W|$. Consequently, the solution is $\frac{1}{2}$ BPS within an appropriate $\mathcal{N}=2$ truncation.

When $\mathfrak{H}^{*}$ is semi-simple, on the other hand, the BPS parameter $c$ cannot be expressed in the simple form (1.2), but is a more complicated non-rational function of the physical charges. In this case, the condition $c=0$ does not necessarily imply saturation of any BPS bound. This is borne out by Papapetrou-Majumdar type solutions in the second category. As shown in [8] this can happen in particular for maximal $\mathcal{N}=8$ supergravity, or more generally for theories that can be obtained by dimensional reduction of five-dimensional supergravities on a circle. As we will show, such theories with $n$ vector fields in four dimensions admit multi-black hole solutions depending on $1+n$ harmonic functions ( $n$ for asymptotically Minkowski solutions) that do not define $\frac{1}{2}$ BPS solutions of any $\mathcal{N}=2$ truncation of the theory.

Because they rely essentially only on properties of the three-dimensional duality groups $\mathfrak{G}$, our results in principle allow a general classification. In order to bring out the main points as clearly as possible, we here restrict attention mostly to the example of maximal $\mathcal{N}=8$ supergravity [12]. The $\mathcal{N}=4$ supergravities will be discussed in detail in a companion publication [13]. The extremal spherically symmetric black holes of maximal supergravity can be understood within the simplified context of the so-called $S T U \mathcal{N}=2$ supergravity [14]. From the point of view of the latter, there are three types of generic extremal spherically symmetric black holes: the $\frac{1}{2}$ BPS ones, the non-BPS ones for which the central charge vanishes on the horizon $(Z=0)$, and the non-BPS ones for which the central charge does not vanish on the horizon $(Z \neq 0)$ $[15,16]$. Both $\frac{1}{2}$ BPS and non-BPS black holes for which the central charge vanishes on the horizon correspond to $\frac{1}{8}$ BPS black holes within maximal supergravity. Within the $S T U$ model such solutions are related by exchanging the vector fields belonging to the gravity multiplet with those in the matter multiplets, respectively. By contrast, the non-BPS solutions with $Z \neq 0$ are still non-BPS when embedded in maximal supergravity. As we will explain in this paper, such solutions are associated to theories that define compactification on a circle of theories defined in $4+1$ dimensions. For instance, pure gravity in five dimensions dimensionally reduced on a circle does admit extremal solutions, and it obviously defines a consistent truncation of any theory originating from five dimensions [11]. One then has a corresponding $\mathfrak{H}^{*}$-orbit of 
extremal solutions, which for the STU model corresponds to the non-BPS solutions with non-vanishing central charge on the horizon.

The plan of the paper is as follows. We first review the general formalism [6,8] and explain how the Papapetrou-Majumdar solutions can be read from the nilpotent orbits. Then we explain how one can understand the two categories of Papapetrou-Majumdar solutions within this formalism. We display in detail the spectrum of such solutions in maximal supergravity. In a subsequent publication by one of the authors [13], the case of $\mathcal{N}=4$ supergravity coupled to $n$ vector multiplets will be discussed in detail. In particular, it will include explicit non-BPS multi-black hole solutions belonging to the second category (the would be non-BPS solutions with $Z \neq 0$ within the $S T U$ model) depending on $6+n$ independent harmonic functions.

\section{Extremal solutions and nilpotent orbits}

Stationary solutions of Einstein theory of gravitation in four dimensions coupled to abelian vector fields and scalar fields parametrising a symmetric space $\mathfrak{G}_{4} / \mathfrak{H}_{4}$ can be effectively obtained by solving the equations of motion of an associated non-linear sigma model coupled to gravity in three spatial dimensions (i.e. with Euclidean signature). When the isometry group $\mathfrak{G}_{4}$ acts on the abelian vector fields in a faithful representation $\mathfrak{l}_{4}$ to define a non-linear symmetry of the equations of motion, one thus arrives at a three-dimensional non-linear sigma which unifies all the physical bosonic degrees of freedom into a single pseudo-Riemannian coset space $\mathfrak{G} / \mathfrak{H}^{*}$, with $\mathfrak{G}$ a simple Lie group and $\mathfrak{H}^{*}$ a non-compact real form of its maximal compact subgroup $[6,7]$.

To derive the equations of motion for a coset representative $\mathcal{V}$ in $\mathfrak{G} / \mathfrak{H}^{*}$, one decomposes the Maurer-Cartan form $\mathcal{V}^{-1} d \mathcal{V}$ into its coset and its $\mathfrak{h}^{*}$ components,

$$
\mathcal{V}^{-1} d \mathcal{V}=Q+P, \quad Q \equiv Q_{\mu} d x^{\mu} \in \mathfrak{h}^{*}, \quad P \equiv P_{\mu} d x^{\mu} \in \mathfrak{g} \ominus \mathfrak{h}^{*}
$$

where $\mu, v, \cdots=1,2,3$. The equation of motion for the scalars reads

$$
d \star \mathcal{V} P \mathcal{V}^{-1}=0
$$

where $\star$ is the Hodge star operator associated to the three-dimensional Riemannian metric $g_{\mu \nu}$. The Einstein equation is

$$
R_{\mu \nu}=\operatorname{Tr} P_{\mu} P_{\nu}
$$

Because in three dimensions the Riemann tensor is entirely determined by the Ricci tensor, the three-dimensional metric $g_{\mu \nu}$ is flat if the right-hand side of (2.3) vanishes.

Black hole solutions of the four-dimensional theory correspond to instantons of the associated three-dimensional non-linear sigma model over $\mathfrak{G} / \mathfrak{H}^{*}$. The spherically symmetric black holes (including the asymptotically Taub-NUT ones) are entirely 
characterised by their $\mathfrak{G}$-Noether charge

$$
\mathcal{Q} \equiv \frac{1}{4 \pi} \int_{\partial V} \star \mathcal{V} P \mathcal{V}^{-1}
$$

and the asymptotic value of the scalars fields $\mathcal{V}_{0} \in \mathfrak{G}_{4} \subset \mathfrak{G}$ at spatial infinity [6]. Here it will be more convenient to characterise the solutions in term of a modified conserved charge $\mathscr{C}$ obtained by rotating $\mathcal{Q}$ back into the coset

$$
\mathscr{C} \equiv \mathcal{V}_{0}^{-1} \mathcal{Q} \mathcal{V}_{0} \in \mathfrak{g} \ominus \mathfrak{h}^{*}
$$

which we will call the 'Noether charge' for simplicity (this designation being unambiguous since we will never refer to $\mathcal{Q}$ itself).

Any spherically symmetric black hole then admits the following $\mathfrak{G} / \mathfrak{H}^{*}$ representative [17]

$$
\mathcal{V}=\mathcal{V}_{0} \exp \left(\frac{1}{2 c} \ln \left(\frac{r-c}{r+c}\right) \mathscr{C}\right)
$$

for some matrix $\mathscr{C}$, where $r$ is the Weyl radius, associated to the three-dimensional Riemannian metric ${ }^{1}$

$$
g_{\mu \nu} d x^{\mu} d x^{\nu}=d r^{2}+\left(r^{2}-c^{2}\right)\left(d \theta^{2}+\sin ^{2} \theta d \varphi^{2}\right)
$$

The BPS parameter $c$ is defined in terms of the Noether charge as

$$
c^{2} \equiv \frac{1}{k} \operatorname{Tr} \mathscr{C}^{2},
$$

Here $k \equiv \operatorname{Tr} \mathbf{h}^{2}$ is a positive integer associated to the group $\mathfrak{G}$ and the following five-graded decomposition of its Lie algebra $\mathfrak{g}$ with respect to its subalgebra $\mathfrak{g l} \mathfrak{l}_{1} \equiv \mathbb{R} \mathbf{h}$

$$
\mathfrak{g} \cong \mathbf{1}^{(-2)} \oplus \mathfrak{l}_{4}^{(-1)} \oplus\left(\mathfrak{g l}_{1} \oplus \mathfrak{g}_{4}^{(0)}\right) \oplus \mathfrak{l}_{4}^{(1)} \oplus \mathbf{1}^{(2)}
$$

This decomposition shows explicitly how the duality group $\mathfrak{G}_{4}$ of the four-dimensional theory and its representation $\mathfrak{l}_{4}$ are embedded in $\mathfrak{G}$.

Given a solution of the three-dimensional equations of motion one can reconstruct the solution in four dimensions as follows. In order to read off the Kaluza-Klein ansatz for the four-dimensional metric and the abelian vector fields from the above 'data', one must first rotate the coset representative $\mathcal{V}$ into a parabolic (triangular) gauge

\footnotetext{
1 The Weyl radius $r$ is related to the standard radial coordinate $\tilde{r}$ via $\tilde{r}=r+m$ such that, e.g. the horizon of the Schwarzschild solution corresponds to $r=m$ (see, e.g. [18]). Unlike the usual Schwarzschild coordinates the Weyl coordinates are duality invariant, as follows from the duality invariance of the BPS parameter $c$ and the split of the four-metric in (2.15) below.
} 
$\mathcal{V} \in \mathfrak{P}$ where $\mathfrak{P}$ is the maximal parabolic subgroup of $\mathfrak{G}$ defined from the five-graded decomposition (2.9) with Lie algebra $\mathfrak{p}$

$$
\mathfrak{p} \cong\left(\mathfrak{g l}_{1} \oplus \mathfrak{g}_{4}^{(0)}\right) \oplus \mathfrak{l}_{4}^{(1)} \oplus \mathbf{1}^{(2)}
$$

Let us designate by $\ddagger$ the involution that defines $\mathfrak{h}^{*} \subset \mathfrak{g}$, such that $\mathbf{x}-\mathbf{x}^{\ddagger} \in \mathfrak{h}^{*}$. We write $\mathbf{h}$ for the $\mathfrak{g l}_{1}$ generator, and $\mathbf{e}$ and $\mathbf{f}=\mathbf{e}^{\ddagger}$ for the grade 2 and -2 generators, respectively. The representation $\mathfrak{l}_{4}$ always admits a $\mathfrak{G}_{4}$ invariant symplectic form (which defines the Dirac quantisation condition) such that two generators $\mathbf{x}$ and $\mathbf{y}$ of $\mathfrak{r}_{4}^{(1)}$ commute to

$$
[\mathbf{x}, \mathbf{y}]=(\mathrm{x}, \mathrm{y})_{\mathfrak{l}_{4}} \mathbf{e}
$$

Defining $\boldsymbol{\Phi}$ as the electromagnetic scalars valued in $\mathfrak{l}_{4}^{(1)}$ and $v \in \mathfrak{G}_{4} / \mathfrak{H}_{4}$ the fourdimensional scalars fields, one writes down the coset representative $\mathcal{V}$ in the parabolic gauge as

$$
\mathcal{V}=\exp (B \mathbf{e}+\boldsymbol{\Phi}) \exp \left(\frac{1}{2} \ln H \mathbf{h}\right) v
$$

A straightforward computation gives

$$
\begin{aligned}
P= & \frac{1}{2} H^{-1} d H \mathbf{h}+\frac{1}{2} H^{-1}\left(d B-\frac{1}{2}(\Phi, d \Phi)_{\mathfrak{l}_{4}}\right)(\mathbf{e}+\mathbf{f}) \\
& +\frac{1}{2} H^{-\frac{1}{2}} L_{4}\left(v^{-1}\right) d\left(\boldsymbol{\Phi}+\boldsymbol{\Phi}^{\ddagger}\right)+\left(v^{-1} d v\right)_{\mid \mathfrak{g}_{4} \ominus \mathfrak{h}_{4}}
\end{aligned}
$$

where $L_{4}$ is the $\mathfrak{l}_{4}$ representation homomorphism. Substituting these expressions into the equation of motion (2.2) it follows that the axion field $B$ defines the Kaluza-Klein vector $\hat{B}$ through

$$
H^{-2} \star\left(d B-\frac{1}{2}(\Phi, d \Phi) \mathfrak{l}_{4}\right)=d \hat{B}
$$

such that the four-dimensional metric reads

$$
d s^{2}=-H\left(d t+\hat{B}_{\mu} d x^{\mu}\right)^{2}+H^{-1} g_{\mu \nu} d x^{\mu} d x^{\nu}
$$

In order to reconstruct the four-dimensional vector fields

$$
\sqrt{2 \pi G} \mathcal{A}=U\left(d t+\hat{B}_{\mu} d x^{\mu}\right)+\hat{A}_{\mu} d x^{\mu}
$$

from (2.13) one must choose a Lagrangian subspace of $\mathfrak{l}_{4}$ with respect to the symplectic form of $\mathfrak{G}_{4}$, such that $\Phi$ splits into $U \oplus A$. After the redefinition $B^{\prime} \equiv B+\frac{1}{2}(U, A)_{\mathfrak{l}_{4}}$, 
the fields $A$ only enter $P$ linearly through their differential $d A$, and can then be dualised to vectors $\hat{A}$ according to their equations of motion.

Let us consider a general non-rotating asymptotically flat solution which does not carry any naked singularity, by which we mean that any singularity is covered by an horizon which is identified with its corresponding Killing horizon (no ergosphere). Such solutions are spherically symmetric black holes [6]. From the point of view of the three-dimensional Riemannian space $V$, the horizon $\mathscr{H}$ corresponds to the single point $r=c$ on $V$ (cf. (2.7)) with an instanton-like singularity. Because the space-time volume element $H^{-1} \sqrt{g}$ is regular on the horizon of a non-extremal black hole, while $H$ goes to zero there, the three-dimensional volume element $\sqrt{g}$ vanishes on the horizon. It follows that all components of $\mathcal{V}$ must tend to the same value as $r \rightarrow c$ and are thus constant on $\mathscr{H}$. Using the fact that the dependency on $\hat{B}$ can be neglected on $\mathscr{H}$, the expression of the horizon area $A_{\mathscr{H}}$ in the coordinates (2.7)

$$
A_{\mathscr{H}} \equiv \int_{\mathscr{H}}\left(r^{2}-c^{2}\right) H^{-1} \sin \theta d \theta \wedge d \varphi
$$

shows that for $A_{\mathscr{H}}>0, H$ behaves like

$$
H=\frac{4 \pi}{A_{\mathscr{H}}}\left(r^{2}-c^{2}\right)+\mathcal{O}\left(\left(r^{2}-c^{2}\right)^{\frac{3}{2}}\right)
$$

near $\mathscr{H}$. Similarly, the surface gravity $\kappa$ can be computed from the Killing vector $\xi^{M} \partial_{M} \equiv \partial_{t}$ as

$$
\kappa^{2} \equiv-\frac{1}{2} \lim _{r \rightarrow c} \nabla^{M} \xi^{N} \nabla_{M} \xi_{N}=\frac{1}{4}\left[\partial^{\mu} H \partial_{\mu} H-H^{4} \partial^{\mu} \hat{B}^{v}\left(\partial_{\mu} \hat{B}_{\nu}-\partial_{\nu} \hat{B}_{\mu}\right)\right]_{\mathscr{H}}
$$

Exploiting the fact that $\hat{B}$ is regular on the horizon we thus obtain

$$
\kappa=\left.\frac{1}{2} \partial_{r} H\right|_{\mathscr{H}}=\frac{4 \pi c}{A_{\mathscr{H}}}
$$

Similarly, using the behaviour of the fields near the horizon, one computes that the charge associated to the horizon is given by

$$
\mathscr{C} \equiv \frac{1}{4 \pi} \mathcal{V}_{0}^{-1} \int_{\mathscr{H}} \star \mathcal{V} P \mathcal{V}^{-1} \mathcal{V}_{0}=\lim _{r \rightarrow c}\left(\left(r^{2}-c^{2}\right) \mathcal{V}_{0}^{-1} \mathcal{V} P_{r} \mathcal{V}^{-1} \mathcal{V}_{0}\right)
$$

It follows that

$$
\operatorname{Tr} \mathscr{C}^{2}=\lim _{r \rightarrow c}\left(\left(r^{2}-c^{2}\right)^{2} \operatorname{Tr} P_{r}^{2}\right)
$$


Using the behaviour of $H$ near the horizon, the equation of motion (2.14) and the assumption that all the fields $\hat{B}, \Phi$ and $v$ are regular on the horizon, we arrive at

$$
\left(r^{2}-c^{2}\right) P_{r}=c \mathbf{h}+\mathcal{O}\left(\sqrt{r^{2}-c^{2}}\right)
$$

recovering the formula (2.8) as expected. Therefore, we obtain that the product of the horizon area and the surface gravity of a non-rotating black hole is determined by the Killing norm of the $\mathfrak{G}$-Noether charge as

$$
A_{\mathscr{H}} \kappa=4 \pi c
$$

As a result, non-rotating extremal solutions, for which $\kappa=0$, carry a Noether charge satisfying $\operatorname{Tr} \mathscr{C}^{2}=0$ (since $\kappa$ is identified with the temperature of the black hole in the thermodynamic interpretation, it follows that all such solutions are characterised by zero temperature). Therefore, from (2.7) we see that spherically symmetric extremal black holes solutions can be viewed as instantons over flat three-dimensional space, with the Euclidean three-dimensional metric $g_{\mu \nu}=\delta_{\mu \nu}$. For these solutions the coset representative $\mathcal{V}$ takes the form

$$
\mathcal{V}=\mathcal{V}_{0} \exp \left(-\frac{1}{r} \mathscr{C}\right)
$$

with a Noether charge satisfying $\operatorname{Tr} \mathscr{C}^{2}=0$. Inspection of the equations of motion shows that $\frac{1}{r}$ can be replaced by any solution of the three-dimensional Laplace equation $\Delta \mathcal{H}=0$. Such solutions were first discovered by Papapetrou and Majumdar in Maxwell-Einstein theory [1,2]. The main purpose of this paper is to study these solutions in a systematic manner by exploiting the maximal duality symmetry $\mathfrak{G}$ acting on them.

\section{Solutions of Papapetrou-Majumdar type}

To obtain the solutions of Papapetrou-Majumdar type, we replace the formula (2.25) by the general Ansatz for the coset representative $\mathcal{V}$

$$
\mathcal{V}(x)=\mathcal{V}_{0} \exp \left(-\sum_{n} \mathcal{H}^{n}(x) \mathscr{C}_{n}\right)
$$

with Lie algebra elements $\mathscr{C}_{n} \in \mathfrak{g} \ominus \mathfrak{h}^{*}$, and where the functions $\mathcal{H}^{n}(x)$ are to be determined from the equations of motion. It is straightforward to see that $P$ depends linearly on the functions $\mathcal{H}^{n}$ if and only if [9]

$$
\left[\mathscr{C}_{m},\left[\mathscr{C}_{n}, \mathscr{C}_{p}\right]\right]=0
$$


Under this assumption, one obtains that

$$
P=\sum_{n} d \mathcal{H}^{n} \mathscr{C}_{n}, \quad Q=\frac{1}{2} \sum_{n m} \mathcal{H}^{n} d \mathcal{H}^{m}\left[\mathscr{C}_{n}, \mathscr{C}_{m}\right]
$$

and the equations of motion reduce to

$$
R_{\mu \nu}=\sum_{m n} \partial_{\mu} \mathcal{H}^{m} \partial_{\nu} \mathcal{H}^{n} \operatorname{Tr} \mathscr{C}_{m} \mathscr{C}_{n}, \quad d \star d \mathcal{H}^{n}=0
$$

For the equations of motion to be linear in $\mathcal{H}^{n}$, one must require in addition that

$$
\operatorname{Tr} \mathscr{C}_{m} \mathscr{C}_{n}=0
$$

Any set of matrices satisfying (3.2) and (3.5) yields a solution of the theory with a flat three-dimensional metric, if the $\mathcal{H}^{n}$ are arbitrary harmonic functions. Therefore the problem of solving the three-dimensional field equations can be reduced to solving the algebraic equations (3.2) and (3.5). The main new insight from the present analysis (and from [8]) is that this problem, in turn, can be reduced to the construction and classification of the nilpotent orbits of $\mathfrak{g}$ via their corresponding normal triplets (see below) and the associated graded decompositions of $\mathfrak{g}$, as we shall now explain.

Any element of a Lie algebra $\mathfrak{g}$ can be written as the sum of a diagonalisable element and a nilpotent element. There is thus no loss of generality in assuming that each $\mathscr{C}_{n}$ in the general ansatz (3.1) is either nilpotent or diagonalisable. Two elements $\mathscr{C}_{1}$ and $\mathscr{C}_{2}$ satisfying Eq. (3.2) either commute, or generate a Heisenberg subalgebra of $\mathfrak{g}$. From the representation theory of Heisenberg algebras it follows that any linear combination of $\mathscr{C}_{1}$ and $\mathscr{C}_{2}$ is nilpotent in the latter case. Therefore the matrices $\mathscr{C}_{n}$ are either diagonalisable elements commuting with all the others, or nilpotent elements such that any linear combination of them is likewise nilpotent.

The set of nilpotent elements of $\mathfrak{g}$ is known to decompose into finitely many $\mathfrak{G}$-orbits, the so-called nilpotent orbits of $\mathfrak{g}$ [19]. For a given nilpotent orbit, one can associate to a given representative $\mathbf{E} \in \mathfrak{g}$ of the orbit, an $\mathfrak{s l}_{2}$ triplet $(\mathbf{H}, \mathbf{E}, \mathbf{F})$ such that [19]

$$
[\mathbf{H}, \mathbf{E}]=2 \mathbf{E} \quad[\mathbf{H}, \mathbf{F}]=-2 \mathbf{F} \quad[\mathbf{E}, \mathbf{F}]=\mathbf{H}
$$

Decomposing the Lie algebra with respect with the eigenvalues of $\mathbf{H}$, one gets a $(2 n+1)$-graded decomposition of $\mathfrak{g}\left(\right.$ where $\left.\mathfrak{g l}_{1} \equiv \mathbb{R} \mathbf{H}\right)$

$$
\mathfrak{g} \cong \mathfrak{g}^{(-n)} \oplus \cdots \oplus \mathfrak{g}^{(-2)} \oplus \mathfrak{g}^{(-1)} \oplus\left(\mathfrak{g l}_{1} \oplus \mathfrak{g}^{(0)}\right) \oplus \mathfrak{g}^{(1)} \oplus \mathfrak{g}^{(2)} \oplus \cdots \oplus \mathfrak{g}^{(n)}
$$

such that $\mathfrak{g}^{(0)}$ is a reductive Lie algebra (i.e. the direct sum of a semi-simple and an abelian algebra) which contains a Cartan subalgebra of $\mathfrak{g}$. From Eq. (3.6), it follows that the nilpotent element $\mathbf{E}$ lies in $\mathfrak{g}^{(2)}$, and the nilpotency degree of $\mathbf{E}$ in the adjoint 
representation ${ }^{2}$ is the lowest integer strictly greater than $n$. The nilpotency degree then follows directly from the grading and is not altered by the detailed commutation rules of the Lie algebra in this graded decomposition. For a general nilpotent orbit, $n$ can be pretty large, but if we consider a nilpotent orbits for which $n \leq 5$, it follows from the graded decomposition that any element $\mathscr{C}_{n} \in \bigoplus_{p=2}^{n} \mathfrak{g}^{(p)}$ satisfies both Eqs. (3.2) and (3.5). There are four distinguished cases, namely

$$
\begin{aligned}
& \operatorname{ad}_{\mathbf{E}}^{6}=0 \Rightarrow n=5 \\
& \operatorname{ad}_{\mathbf{E}}{ }^{5}=0 \Rightarrow n=4 \\
& \operatorname{ad}_{\mathbf{E}}{ }^{4}=0 \Rightarrow n=3 \\
& \operatorname{ad}_{\mathbf{E}}{ }^{3}=0 \Rightarrow n=2
\end{aligned}
$$

All of the above relations also hold for the complexification $\mathfrak{G}_{\mathbb{C}}$ of the duality group $\mathfrak{G}$ and its associated complex Lie algebra $\mathfrak{g}_{\mathbb{C}}$. What is important is that the complex nilpotent orbits in $\mathfrak{g}_{\mathbb{C}}$ are uniquely determined by the nilpotency degree (in several representations), and equivalently by the graded decomposition (3.7). When descending from the complex to the real Lie algebra, the complex nilpotent orbit may decompose into several real orbits, which are the ones relevant for black hole solutions.

The isotropy subalgebra of a representative $\mathbf{E}$ of a given orbit is a subalgebra of $\bigoplus_{p=0}^{n} \mathfrak{g}^{(p)}$. Any solution to Eq. (3.2) can thus be associated to a given $2 n+1$ graded decomposition (3.7) with $n \leq 5$, such that the nilpotents elements lie in $\bigoplus_{p=2}^{n} \mathfrak{g}^{(p)}$ and the diagonalisable elements commute with all the others and lye in $\mathfrak{g}^{(0)}$. Equation (3.5) is trivially satisfied for the nilpotent elements, but strongly constrains the diagonalisable elements of $\mathfrak{g}^{(0)}$.

More specifically, in black hole physics, we are interested in elements which lie in $\mathfrak{g} \ominus \mathfrak{h}^{*}$ in order for the coset representative $\mathcal{V}$ to be in the symmetric gauge. In fact, one can show that there is no loss of generality by doing so, because any more general coset element can be rotated back to this form by a right $\mathfrak{H}^{*}$ gauge transformation. When the $\mathfrak{G}$-orbit of the nilpotent element $\mathbf{E}$ has a non-trivial intersection with $\mathfrak{g} \ominus \mathfrak{h}^{*}$, the triplet can be chosen in such a way that both $\mathbf{E}$ and $\mathbf{F}$ lie in $\mathfrak{g} \ominus \mathfrak{h}^{*}$, and such that $\mathbf{H}$ lies in $\mathfrak{h}^{*}$. It follows then that $\mathfrak{h}^{*}$ decomposes in a similar way as

$$
\mathfrak{h}^{*} \cong \mathfrak{h}^{(-n)} \oplus \cdots \oplus \mathfrak{h}^{(-2)} \oplus \mathfrak{h}^{(-1)} \oplus\left(\mathfrak{g l}_{1} \oplus \mathfrak{h}^{(0)}\right) \oplus \mathfrak{h}^{(1)} \oplus \mathfrak{h}^{(2)} \oplus \cdots \oplus \mathfrak{h}^{(n)}
$$

such that $\mathfrak{h}^{(0)}$ is a reductive Lie algebra which contains a Cartan subalgebra of $\mathfrak{h}^{*}$, and $\mathbf{E} \in \mathfrak{g}^{(2)} \ominus \mathfrak{h}^{(2)}$. The elements of $\mathfrak{g}^{(p)} \ominus \mathfrak{h}^{(p)}$ (for $p \geq 2$ ) generate a nilpotent Lie algebra $\mathfrak{n}^{(p)}$, which decomposes as (for $n \leq 5$ )

$$
\mathfrak{n}^{(p)} \cong\left(\mathfrak{g}^{(p)} \ominus \mathfrak{h}^{(p)}\right) \oplus \mathfrak{h}^{(2 p)}
$$

Any set of charge matrices $\mathscr{C}_{n}$ lying in $\bigoplus_{p=2}^{n}\left(\mathfrak{g}^{(p)} \ominus \mathfrak{h}^{(p)}\right)$ therefore satisfies the commutation relations (4.18) and defines a multi-black hole solution via the Ansatz (3.1).

2 Which is defined to be the smallest integer $k$ such that $\left(\operatorname{ad}_{\mathbf{E}}\right)^{k}(\mathbf{x})=0$ for all $\mathbf{x} \in \mathfrak{g}$. 
In the case of the graded decomposition (2.9), which will be the one of interest as we are going to see, the isotropy subgroups of grade zero $\mathfrak{J}_{n}^{(0)} \subset \mathfrak{G}$ and $\mathfrak{K}_{n}^{(0)} \subset \mathfrak{H}^{*}$ of a generic element $\mathbf{E} \in \mathfrak{l}_{4}^{(2)} \ominus \mathfrak{h}^{(2)}$ coincide with the isotropy subgroups of $\mathfrak{G}_{4}$ and $\mathfrak{H}_{4}$ of the corresponding electromagnetic charges, respectively. These subgroups also define the so-called moduli spaces of black hole attractors as $\mathfrak{J}_{n}^{(0)} / \mathfrak{K}_{n}^{(0)} \subset \mathfrak{G}_{4} / \mathfrak{H}_{4}$ [14]. The Cartan norm is thus positive definite on $\mathfrak{j}_{n}^{(0)} \ominus \mathfrak{k}_{n}^{(0)}$ and there is no diagonalisable element to consider in the solutions of $(3.2,3.5)$.

Let us next consider a more general Ansatz than (3.1) including Lie algebra elements $\mathscr{A}_{s} \in \bigoplus_{p=2}^{n} \mathfrak{h}^{(n)}$. From the grading and the Campbell-Hausdorff formula it follows that

$$
\begin{aligned}
\mathcal{V} & =\mathcal{V}_{0} \exp \left(-\sum_{n} \mathcal{H}^{n} \mathscr{C}_{n}-\sum_{s} \mathcal{K}^{s} \mathscr{A}_{s}\right) \\
& =\mathcal{V}_{0} \exp \left(-\sum_{n} \mathcal{H}^{n} \mathscr{C}_{n}+\frac{1}{2} \sum_{s n} \mathcal{K}^{s} \mathcal{H}^{n}\left[\mathscr{A}_{s}, \mathscr{C}_{n}\right]\right) \exp \left(-\sum_{s} \mathcal{K}^{s} \mathscr{A}_{s}\right)
\end{aligned}
$$

such that this Ansatz is in fact equivalent to (3.1) up to a right $\mathfrak{H}^{*}$ gauge transformation. $^{3}$

The solutions obtained by the above construction may in principle exhibit naked singularities. Quite generally, the latter can be of two types. For solutions not obeying any 'no force' property, there are usually rod-like singularities which appear in static multi-black hole solutions in order to balance the gravitational pull between any two black holes, and which manifest themselves as string-like singularities of the Riemannian metric $g_{\mu \nu}$. For example, in the axisymmetric case, ${ }^{4}$ they appear in Weyl coordinates

$$
g_{\mu \nu} d x^{\mu} d x^{\nu}=e^{2 \sigma}\left(d z^{2}+d \rho^{2}\right)+\rho^{2} d \varphi^{2}
$$

if the function $\sigma(\rho, z)$ does not vanish in the limit $\rho \rightarrow 0$ on the axis in between two interacting black holes. This type of singularity cannot occur for the solutions built on the conditions (3.2) and (3.5) because the three-dimensional metric is then flat and regular everywhere by (2.3). Consequently, the singularities of the four-dimensional theory solely originate from the singularities of the scalar fields which are located at the poles of the harmonic functions $\mathcal{H}^{n}$. Naked singularities of such solutions are thus entirely due to the individual black holes themselves, and can be avoided by choosing each charge $\mathscr{C}_{n}$ so that the individual black holes have their singularities covered by horizons. This way one makes sure that each pole of the harmonic functions corresponds to an horizon in four dimensions.

It is commonly assumed that all the spherically symmetric extremal black holes without naked singularities correspond to particular limits of non-extremal spherically

\footnotetext{
3 Note that $\left[\mathscr{A}_{s}, \mathscr{C}_{n}\right] \in \bigoplus_{p=4}^{n}\left(\mathfrak{g}^{(p)} \ominus \mathfrak{h}^{(p)}\right)$.

4 In which case exact solutions with several black holes that do not share any no force property are known explicitly [18].
} 
symmetric black holes which do not carry any naked singularity themselves. Within the class of model discussed in this paper, the asymptotically flat regular non-extremal solutions are all in the $\mathfrak{H}^{*}$-orbit of uncharged Kerr-solutions with scalar fields having any constant value [6]. It follows that the corresponding Noether charge $\mathscr{C}$ satisfies a cubic characteristic equation [8]

$$
\mathscr{C}^{3}=c^{2} \mathscr{C}
$$

in the fundamental representation (which is the spinor representation of its doublecover when $\mathfrak{G}$ is an orthogonal group), save for two particular cases for which $\mathfrak{G}$ is a non-compact real form of $E_{8}$, corresponding to maximal $\mathcal{N}=8$ supergravity and the 'magic' $\mathcal{N}=2$ supergravity associated to the octonions [20]. In these two cases the characteristic equation is quintic [8], and must be satisfied in the $\mathbf{3 8 7 5}$ representation that appears in the symmetric product of two copies of the adjoint representation,

$$
\mathscr{C}^{5}=5 c^{2} \mathscr{C}^{3}-4 c^{4} \mathscr{C}
$$

It follows that regular extremal solutions (for which $c=0$ ) carry a nilpotent Noether charge $\mathscr{C}$, which vanishes at the third power in the fundamental representation, i.e. $\mathscr{C}^{3}=0$, in the generic case, or at the fifth power in the $\mathbf{3 8 7 5}$ representation of $E_{8}$ when $\mathfrak{G}$ is a real form of $E_{8}$ [8]. So as a first consequence, one can restrict attention to nilpotent elements $\mathscr{C}_{n}$ in considering regular multi-black holes, because any diagonalisable elements of vanishing Killing norm would lead to naked singularities.

The nilpotency conditions are preserved by the action of the complexified group $\mathfrak{G}_{\mathbb{C}}$, and they determine one single complex nilpotent orbit with representative $\mathbf{E}_{5}$. As already mentioned the complex orbit may decompose into several real nilpotent orbits when one descends to the real group $\mathfrak{G} \subset \mathfrak{G}_{\mathbb{C}}$. Acting with the complex Lie group $\mathfrak{G}_{\mathbb{C}}$ on the representative element $\mathbf{E}_{5}$ we obtain a dense open subset within the complex variety defined by the nilpotency condition [19]. Therefore, any solution $\mathscr{C}$ of the characteristic equation (3.13) or (3.14) lies in the closure of this complex orbit inside the variety of nilpotent elements of $\mathfrak{g}_{\mathbb{C}}$. Since, in addition we require this orbit to be real and to have a non-trivial intersection with $\mathfrak{g} \ominus \mathfrak{h}^{*}$, we have

$$
\mathscr{C} \in \overline{\mathfrak{G}_{\mathbb{C}} \cdot \mathbf{E}_{5}} \cap \mathfrak{g} \ominus \mathfrak{h}^{*}
$$

It turns out that the $\mathfrak{s l}_{2}(\mathbb{C})$ triplet associated to $\mathbf{E}_{5}$ is such that $\mathbf{H}_{5}=\left[\mathbf{E}_{5}, \mathbf{F}_{5}\right]$ satisfies the same characteristic equation as $2 \mathbf{h}$, and the graded decomposition of $\mathfrak{g}_{\mathbb{C}}$ associated to such nilpotent element is therefore the (complexified) five-graded decomposition associated to the dimensional reduction (2.9). The five-graded decomposition of $\mathfrak{g}_{\mathbb{C}}$ with respect to $\mathbf{H}_{5}$ corresponds to a unique five-graded decomposition of $\mathfrak{g}{ }^{5}$

$$
\mathfrak{g} \cong \mathbf{1}^{(-4)} \oplus \mathfrak{l}_{4}^{(-2)} \oplus\left(\mathfrak{g l} \mathfrak{l}_{1} \oplus \mathfrak{g}_{4}^{(0)}\right) \oplus \mathfrak{l}_{4}^{(2)} \oplus \mathbf{1}^{(4)}
$$

\footnotetext{
5 Although it can be degenerate, as for example for $\mathfrak{e}_{6(-14)}$ and $\mathfrak{e}_{7(-25)}$. Nevertheless the orbit we are interested in is unique in these cases.
} 
In general, the elements $\mathbf{E}$ and $\mathbf{H}$ of a normal triplet do not satisfy the same characteristic equation, and this property is very particular to $\mathbf{h}$ and its five-graded decomposition. In fact, this five-graded decomposition characterises both the minimal semi-simple

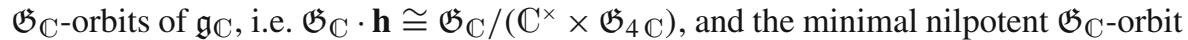
of $\mathfrak{g}_{\mathbb{C}}$, i.e. $\mathfrak{G}_{\mathbb{C}} \cdot \mathbf{e} \cong \mathfrak{G}_{\mathbb{C}} /\left(\mathfrak{G}_{4} \mathbb{C} \ltimes\left(\mathfrak{l}_{4 \mathbb{C}}^{(1)} \oplus \mathbb{C}^{(2)}\right)\right)$.

In order to correspond to a regular solution, the Noether charge $\mathscr{C}$ must lie inside the closure of the $\mathfrak{H}^{*}$-orbits inside $\mathfrak{G}_{\mathbb{C}} \cdot \mathbf{E}_{5} \cap \mathfrak{g} \ominus \mathfrak{h}^{*}$, whose isotropy subgroups are contracted forms of $\mathfrak{H}_{4}$ [8], since the latter is the isotropy subgroup of the Kerr solutions. $\mathfrak{H}_{4}$ being compact, the semi-simple component of the isotropy subgroup of $\mathfrak{H}^{*}$ of such charges is also compact. The most general solutions of Papapetrou-Majumdar type correspond to linear combination of such nilpotent charges which vanish at the sixth power in the adjoint representation. When considering a nilpotent element within the graded decomposition associated to a higher order orbit, its isotropy subgroup generally involves components of negative grading such that the semi-simple component of its isotropy subgroup appears itself through a graded decomposition embedded inside the graded decomposition of $\mathfrak{h}^{*}$ associated to the higher order orbit. It follows that the latter isotropy subgroup cannot be a contracted form of $\mathfrak{H}_{4}$. The relevant linear combinations of charges therefore all lye in the closure of the $\mathfrak{H}^{*}$ orbits of $\mathfrak{G}_{\mathbb{C}} \cdot \mathbf{E}_{5} \cap \mathfrak{g} \ominus \mathfrak{h}^{*}$. We will prove explicitly in [13] that this is indeed the case within $\mathcal{N}=4$ supergravity coupled to $n$ vector multiplets. The only nilpotent orbits we have to consider in order to obtain the most general solutions of Papapetrou-Majumdar type are thus the real nilpotent orbits of $\mathfrak{g}$ of dimension $2 \operatorname{dim}\left[\mathfrak{l}_{4}\right]+2$ associated to the five-graded decomposition (3.16), or more precisely, the $\operatorname{dim}\left[\mathfrak{l}_{4}\right]+1$ dimensional $\mathfrak{H}^{*}$-orbits of nilpotent elements inside $\mathfrak{g} \ominus \mathfrak{h}^{*}$ associated to this five-graded decomposition, such that the $\mathfrak{g l}_{1}$ subalgebra lies in $\mathfrak{h}^{*}$. In general, there are different inequivalent embeddings of $\mathfrak{h}^{*} \subset \mathfrak{g}$ consistent with the graded decomposition (3.16),

$$
\mathfrak{h}^{*} \cong \mathfrak{h}^{(-4)} \oplus \mathfrak{h}^{(-2)} \oplus \mathfrak{g l}_{1} \oplus \mathfrak{h}^{(0)} \oplus \mathfrak{h}^{(2)} \oplus \mathfrak{h}^{(4)}
$$

which correspond to the different $\mathfrak{H}^{*}$-orbits inside $\mathfrak{G}_{\mathbb{C}} \cdot \mathbf{E}_{5} \cap \mathfrak{g} \ominus \mathfrak{h}^{*}$. There are inequivalent Papapetrou-Majumdar solutions associated to each of those graded decompositions, whose number of independent harmonic functions are $\operatorname{dim}\left[\mathfrak{l}_{4}^{(2)} \ominus \mathfrak{h}^{(2)}\right]+1-$ $\operatorname{dim}\left[\mathfrak{h}^{(4)}\right]$.

\section{Supersymmetric solutions}

When the theory coincides with the bosonic sector of a supergravity theory, it is well known that BPS black holes preserving identical supersymmetry charges define multiparticle solutions of Papapetrou-Majumdar type. We are now going to see how this can be understood in the general framework developed in the foregoing section. To this aim we recall a main result of [8], namely the fact that the Noether charge admits a dual description, either as a matrix $\mathscr{C}$ valued in $\mathfrak{g} \ominus \mathfrak{h}^{*}$ as in (2.5), or alternatively, and equivalently, as a state $|\mathscr{C}\rangle$ in a fermionic Fock space, transforming in the same representation as the coset matrix. In the latter description the BPS parameter (2.8) is simply given by the indefinite 'norm' of this state, to wit 


$$
c^{2}=\langle\mathscr{C} \mid \mathscr{C}\rangle
$$

For $\mathcal{N}$-extended supergravity, the non-compact group $\mathfrak{H}^{*}$ is the product of the chiral component ${ }^{6}$ of the R-symmetry group $\operatorname{Spin}^{*}(2 \mathcal{N})$ and a group $\mathfrak{H}_{0}^{*}$ depending on the matter content of the theory. The supersymmetry parameters are $S U(2)$ spinors valued in the pseudo-real vector representation of $S O^{*}(2 \mathcal{N})$ which satisfy an $S O^{*}(2 \mathcal{N})$-Majorana condition associated to the pseudo-anti-involutions of $S U(2)$ and $S O^{*}(2 \mathcal{N})$. Using $U(\mathcal{N}) \subset S O^{*}(2 \mathcal{N})$ covariant notations, the spinor parameters are complex $S U$ (2) spinors $\epsilon_{\alpha}^{i}$ valued in the fundamental representation of $U(\mathcal{N})$, with complex conjugates $\epsilon_{i}^{\alpha}$. In order for the solution to be supersymmetric, the corresponding Noether charge state must satisfy the 'Dirac equation' [8]

$$
\left(\epsilon_{\alpha}^{i} a_{i}+\varepsilon_{\alpha \beta} \epsilon_{i}^{\beta} a^{i}\right)|\mathscr{C}\rangle=0
$$

where $a^{i}$ and $a_{j}$ (for $i, j, \cdots=1, \ldots, \mathcal{N}$ ) are the fermionic oscillators from which the spinor representations of $S O^{*}(2 \mathcal{N})$ are built (see appendix B of [8] for details). The condition (4.2) is derived by considering the supersymmetry variation of the dilatino fields in the asymptotic region. Observe that it contains more detailed information than the gravitino variation, which simply yields the necessary condition $c=0$ for Killing spinors to exist (the latter statement also follows from (4.2) as an integrability condition). In searching for extremal solutions, we can thus replace the problem of solving the cubic or quintic nilpotency conditions for the charge matrix $\mathscr{C}$ by the simpler linear condition (4.2) for the associated charge state $|\mathscr{C}\rangle$ [8].

Solutions of (4.2) are characterised by the number of supersymmetries and by their isotropy groups. Generally, the latter are always parabolic subgroups of $\mathfrak{H}^{*}$ because the charge state $|\mathscr{C}\rangle$ is a null vector (i.e. a zero norm state) for $c=0$ by (4.1). In [8] we showed that solving the characteristic equations (3.13) or (3.14) for regular solutions gives rise to a stratified moduli space

$$
\mathcal{M}=\mathcal{M}_{0} \cup \mathcal{M}_{1} \cup \cdots \cup \mathcal{M}_{n}
$$

where the main stratum $\mathcal{M}_{0}$ corresponds to non-extremal solutions (that is, $c^{2}>0$ ), while the remaining strata correspond to solutions with $c=0$ and contain the supersymmetric or BPS solutions as a subspace. When the solution is left invariant by $4 n$ supersymmetry generators, one speaks of an ' $\frac{n}{\mathcal{N}}$ - BPS solution'. In this case a basis of supersymmetry generators can be chosen with spinor parameters $\epsilon_{\alpha}^{A}$, with $A$ running from 1 to $2 n$, which satisfy the reality condition (alias symplectic Majorana condition)

$$
\epsilon_{\alpha}^{A}+\varepsilon_{\alpha \beta} \Omega^{A B} \epsilon_{B}^{\beta}=0
$$

\footnotetext{
${ }^{6}$ By chiral component of $\operatorname{Spin}^{*}(2 \mathcal{N})$, we mean its subgroup $\operatorname{Spin}^{*}(2 \mathcal{N}) / \operatorname{ker}\left(S_{+}\right)$that acts faithfully in the chiral Weyl spinor representation $S_{+}$, i.e. $S L(2, \mathbb{R}) \subset \operatorname{Spin}^{*}(4), S O(2,6) \subset \operatorname{Spin}^{*}(8), \operatorname{Spin}^{*}(16) / \mathbb{Z}_{2} \subset$ $\operatorname{Spin}^{*}(16)$ and $\operatorname{Spin}^{*}(2 \mathcal{N})$ itself for odd $\mathcal{N}$.
} 
for a given symplectic form $\Omega_{A B}$ of $\mathbb{C}^{2 n}$, satisfying $\Omega_{A C} \Omega^{B C}=\delta_{A}^{B}$. Using (4.4) the 'Dirac equation' (4.2) can be further simplified to

$$
\left(a_{A}-\Omega_{A B} a^{B}\right)|\mathscr{C}\rangle=0
$$

Let us designate by $\mathfrak{P}_{\frac{n}{\mathcal{N}}}$ the parabolic subgroup of $S O^{*}(2 \mathcal{N})$ that preserves the set of solutions of (4.4), and thus the set of supersymmetry charges leaving invariant a solution of Noether charge $|\mathscr{C}\rangle$ obeying (4.5). To derive $\mathfrak{P}_{\frac{n}{\mathcal{N}}}$, we note that the Lie algebra associated with $\mathfrak{P}_{\frac{n}{\mathcal{N}}}$ is generated by the eigen vectors of positive eigen value with respect to a $\mathfrak{g l}_{1}$ generator $D_{\frac{n}{\mathcal{N}}}$ satisfying $D_{\frac{n}{\mathcal{N}}} \epsilon_{\alpha}^{A}=\epsilon_{\alpha}^{A}$, which reads

$$
D_{\frac{n}{\mathcal{N}}} \equiv \frac{1}{2}\left(\Omega_{A B} a^{A} a^{B}-\Omega^{A B} a_{A} a_{B}\right) \in \mathfrak{s o}^{*}(2 \mathcal{N})
$$

in the harmonic oscillator basis (see [8] for details). Its action on the spinor parameters satisfying (4.4) can be read off from

$$
\left[D_{\frac{n}{\mathcal{N}}}, \epsilon_{\alpha}^{A} a_{A}+\varepsilon_{\alpha \beta} \epsilon_{A}^{\beta} a^{A}\right]=\epsilon_{\alpha}^{A} a_{A}+\varepsilon_{\alpha \beta} \epsilon_{A}^{\beta} a^{A}
$$

$D_{\frac{n}{\mathcal{N}}}$ defines a five-graded decomposition of $\mathfrak{s o}^{*}(2 \mathcal{N})$,

$$
\begin{aligned}
\mathfrak{s o}^{*}(2 \mathcal{N}) \cong & \mathbf{n}(\mathbf{2} \mathbf{n}-\mathbf{1})^{(-2)} \oplus\left(\mathbf{2} \mathbf{n} \otimes \mathbf{2} \mathbf{n}_{\perp}\right)_{\mathbb{R}}^{(-1)} \\
& \left.\oplus\left(\mathfrak{g l}_{1} \oplus \mathfrak{s u}^{*}(2 n) \oplus \mathfrak{s o}^{*}\left(2 n_{\perp}\right)\right)^{(0)} \oplus\left(\mathbf{2} \mathbf{n} \otimes \mathbf{2} \mathbf{n}_{\perp}\right)_{\mathbb{R}}^{(1)} \oplus \overline{\mathbf{n}(\mathbf{2 n}-\mathbf{1}}\right)^{(2)}
\end{aligned}
$$

with $n_{\perp}=\mathcal{N}-2 n$. Indeed, using the identity,

$$
\left[D_{\frac{n}{\mathcal{N}}}, a^{A} \pm \Omega^{A B} a_{B}\right]= \pm\left(a^{A} \pm \Omega^{A B} a_{B}\right)
$$

one concludes that the grade zero subalgebra is generated by

$$
\begin{gathered}
\frac{1}{2} \Lambda_{A}^{B}\left[a^{A}+\Omega^{A C} a_{C}, a_{B}+\Omega_{B D} a^{D}\right] \in \mathfrak{s u}^{*}(2 n)^{(0)} \\
\left.\frac{1}{2} \Lambda_{\bar{A}}{ }_{\bar{B}}{ }^{\bar{A}}, a_{\bar{B}}\right]+\frac{1}{2} \Lambda_{\bar{A} \bar{B}} a^{\bar{A}} a^{\bar{B}}-\frac{1}{2} \Lambda^{\bar{A} \bar{B}} a_{\bar{A}} a_{\bar{B}} \in \mathfrak{s o}^{*}\left(2 n_{\perp}\right)^{(0)}
\end{gathered}
$$

where $\bar{A}$ is a $U\left(n_{\perp}\right)$ index running from $2 n+1$ to $\mathcal{N}$. The nilpotent generators in $\mathfrak{s o}^{*}(2 \mathcal{N})$ of grade \pm 1 and grade \pm 2 are, respectively,

$$
\begin{aligned}
& \Lambda_{A \bar{B}}\left(a^{A} \pm \Omega^{A C} a_{C}\right) a^{\bar{B}}-\Lambda^{A \bar{B}}\left(a_{A} \mp \Omega_{A C} a^{C}\right) a_{\bar{B}} \in\left(\mathbf{2 n} \otimes \mathbf{2} \mathbf{n}_{\perp}\right)_{\mathbb{R}}^{( \pm 1)} \\
& \frac{1}{2} \Lambda_{A B}\left(a^{A} \pm \Omega^{A C} a_{C}\right)\left(a^{B} \pm \Omega^{B D} a_{D}\right)-\frac{1}{2} \Lambda^{A B}\left(a_{A} \mp \Omega_{A C} a^{C}\right)\left(a_{B} \mp \Omega_{B D} a^{D}\right) \\
& \quad \in \mathbf{n}(\mathbf{2 n}-\mathbf{1})^{( \pm 2)}
\end{aligned}
$$


The covariance of the spinor parameter satisfying (4.4) under $\mathfrak{s u}^{*}(2 n)$ follows from the commutation relations

$$
\begin{aligned}
& \left\{a^{A}+\Omega^{A C} a_{C}, a^{B}+\Omega^{B D} a_{D}\right\}=0 \\
& \left\{a^{A}+\Omega^{A C} a_{C}, a_{B}+\Omega_{B D} a^{D}\right\}=2 \delta_{B}^{A}
\end{aligned}
$$

The parabolic subgroup $\mathfrak{P}_{\mathcal{N}} \subset S O^{*}(2 \mathcal{N})$ preserving the set of spinor parameters satisfying (4.4) is thus defined by the Lie algebra

$$
\begin{aligned}
\mathfrak{p}_{\frac{n}{\mathcal{N}}} \cong & \left(\mathfrak{g l}_{1} \oplus \mathfrak{s u}^{*}(2 n) \oplus \mathfrak{s o}^{*}\left(2 n_{\perp}\right)\right)^{(0)} \\
& \oplus\left(\mathbf{2} \mathbf{n} \otimes \mathbf{2} \mathbf{n}_{\perp}\right)_{\mathbb{R}}^{(1)} \oplus \overline{\mathbf{n}(\mathbf{2 n}-\mathbf{1})}{ }^{(2)} \subset \mathfrak{s o}^{*}(2 \mathcal{N})
\end{aligned}
$$

This group preserves the set of spinor parameters (4.4) in the sense that they are only rotated by the subgroup $S U^{*}(2 n)$ and rescaled by $G L(1, \mathbb{R})$, while the remaining generators of $\mathfrak{P}_{\frac{n}{\mathcal{N}}}$ act trivially on them. In conclusion, acting on an $\frac{n}{\mathcal{N}}$ BPS spherically symmetric solution with an element of $\mathfrak{P}_{\frac{n}{\mathcal{N}}} \times \mathfrak{H}_{0}^{*}$, one obtains another $\frac{n}{\mathcal{N}}$ BPS spherically symmetric solution which preserves the same supersymmetry charges.

For asymptotically flat solutions, i.e. asymptotically Taub-NUT space-times, the $\mathfrak{G}$ Noether charge $\mathscr{C}$ is valued in $\mathfrak{g} \ominus \mathfrak{h}^{*}$. In supergravity, this is an irreducible representation of $\operatorname{Spin}^{*}(2 \mathcal{N}) \times \mathfrak{H}_{0}^{*}$, that is, the product of the Weyl spinor representation $\mathcal{S}_{+}$of $\operatorname{Spin}^{*}(2 \mathcal{N})$ and an irreducible representation $\mathcal{R}$ of $\mathfrak{H}_{0}^{*}$, possibly with an extra reality condition.

For simplicity let us first restrict ourselves to $\frac{1}{\mathcal{N}}$ BPS solutions. The theories admitting BPS solutions of higher BPS degree are quite restricted anyway and further relevant examples will be discussed in [13]. In this case the generator $D_{\frac{1}{N}}$ defines the five graded decomposition (which is an example of the decomposition (3.17))

$$
\begin{aligned}
\mathfrak{s o}^{*}(2 \mathcal{N}) \cong & \mathbf{1}^{(-2)} \oplus\left(\mathbf{2} \otimes \mathbf{2} \mathbf{n}_{\perp}\right)_{\mathbb{R}}^{(-1)} \oplus\left(\mathfrak{g l}_{1} \oplus \mathfrak{s u}(2) \oplus \mathfrak{s o}^{*}\left(2 n_{\perp}\right)\right)^{(0)} \\
& \oplus\left(\mathbf{2} \otimes \mathbf{2} \mathbf{n}_{\perp}\right)_{\mathbb{R}}^{(1)} \oplus \mathbf{1}^{(2)}
\end{aligned}
$$

and the Weyl spinor representation of $\operatorname{Spin}^{*}(2 \mathcal{N})$ decomposes accordingly as

$$
\mathcal{S}_{+} \cong S_{+}^{(-1)} \oplus\left(\mathbf{2} \otimes S_{-}\right)^{(0)} \oplus S_{+}^{(1)}
$$

where $S_{ \pm}$are the Weyl spinor representations of $S O^{*}\left(2 n_{\perp}\right)$. Using the fact that the $\frac{1}{\mathcal{N}}$-BPS Killing spinors lie in the grade-one component of the vector representation of $S O^{*}(2 \mathcal{N})$,

$$
\mathbf{2} \mathcal{N} \cong \mathbf{2}^{(-1)} \oplus \mathbf{2 n}_{\perp}^{(0)} \oplus \mathbf{2}^{(1)}
$$

one sees that the solutions of the Dirac equations are simply obtained by taking the charge matrix $\mathscr{C}$ to lie in the grade-one component of $\mathfrak{g} \ominus \mathfrak{h}^{*}$,

$$
\mathscr{C} \in\left(S_{+} \otimes \mathcal{R}\right)^{(1)} \subset \mathcal{S}_{+} \otimes \mathcal{R}
$$


It follows from the graded decomposition of $\mathfrak{g}$ with respect to $D_{\frac{1}{\mathcal{N}}}$ that for any charge matrices $\mathscr{C}_{n} \in\left(S_{+} \otimes \mathcal{R}\right)^{(1)}$,

$$
\left[\mathscr{C}_{n}, \mathscr{C}_{m}\right] \in \mathbf{1}^{(2)} \subset \mathfrak{s o}^{*}(2 \mathcal{N}) \Rightarrow\left[\mathscr{C}_{n},\left[\mathscr{C}_{m}, \mathscr{C}_{p}\right]\right]=0
$$

In this way one obtains that such solution is of the type discussed in the preceding section, with $\mathbf{H}=2 D_{\frac{1}{\mathcal{N}}}$.

For $n \geq 1$, the general solution of (4.5) is obtained by use of the identity

$$
\left(a_{A}-\Omega_{A B} a^{B}\right) \exp \left(\frac{1}{2} \Omega_{C D} a^{C} a^{D}\right)|0\rangle=0
$$

Consequently, if the charge $\mathscr{C}$ is represented by the state

$$
|\mathscr{C}\rangle=\left(\left(W+Z_{i j} a^{i} a^{j}+\cdots\right)|0\rangle,\left(z^{\mathcal{A}}+\Sigma_{i j}^{\mathcal{A}} a^{i} a^{j}+\cdots\right)|0\rangle\right)
$$

the general $\frac{n}{\mathcal{N}}$-BPS solution reads

$$
|\mathscr{C}\rangle=\left(e^{\frac{1}{2} \Omega_{A B} a^{A} a^{B}}\left(W+Z_{\bar{A} \bar{B}} a^{\bar{A}} a^{\bar{B}}\right)|0\rangle, e^{\frac{1}{2} \Omega_{A B} a^{A} a^{B}} z^{\mathcal{A}}|0\rangle\right)
$$

where $\bar{A}$ runs from $2 n+1$ to $\mathcal{N}$, and $\mathcal{A}$ labels the matter multiplets if $\mathcal{N} \leq 4$. An additional reality condition (Majorana-Weyl condition) may have to be imposed on the state if $\mathfrak{g} \ominus \mathfrak{h}^{*}$ admits one. It ensures that the scalar charges are completely determined in function of the others for any solution of the Dirac equation. ${ }^{7}$ In this case, the generator $\mathbf{H}$ is identified as

$$
\mathbf{H}=\frac{2}{n} D_{\frac{n}{\mathcal{N}}}
$$

and $\mathbf{E}$ with the charge matrix $\mathscr{C}$ in such a way that the commutator $[\mathbf{H}, \mathbf{E}]$ corresponds to the action of the operator (4.22) on the state $|\mathscr{C}\rangle .{ }^{8}$ Because the component of grade two (with respect to $D_{\frac{n}{N}}$ ) of $\mathfrak{s o}^{*}\left(2 \mathcal{N}\right.$ ) leaves the $\frac{n}{\mathcal{N}}$-BPS Killing spinors invariant, the Killing equation trivially reduces to $d \epsilon_{\alpha}^{A}=0$, and it follows from (4.5) that the corresponding supersymmetry variation of the dilatino fields vanishes.

Having constructed the appropriate charge matrices $\mathscr{C}$ it is now straightforward to construct multi-black hole solutions of the four-dimensional theory following the procedure described in Sect. 2. If the different matrices $\mathscr{C}_{m}$ satisfy (4.2) with respect to the same supersymmetry parameters, the resulting multi-black hole solution will also be supersymmetric. If the 'constituent' black holes are supersymmetric with respect to different supersymmetries, on the other hand, the multi-black hole configuration will

\footnotetext{
${ }^{7}$ For instance for $\mathcal{N}=2,3$ the scalar charges are manifestly determined in (4.21). For $\mathcal{N}=4$ the complex self-duality condition on the vector multiplets ensures that this is still the case, and similarly for $\mathcal{N}=8$ through the complex-selfduality of the multiplet.

8 That $|\mathscr{C}\rangle$ is always null for BPS states obeying (4.5) then simply follows from $\operatorname{Tr} \mathbf{E}^{2}=0$.
} 
no longer be supersymmetric, and in general then exhibit naked singularities unless the 'constituent' BPS black holes have a vanishing horizon area. Examples of such solutions which are regular are given in [13]. Although supersymmetry is not required for the existence of Papapetrou-Majumdar type solutions, it greatly simplifies their explicit derivation. This is because the algebraic equations determining representatives of nilpotent orbits are not trivial to solve in general, whereas the linear Dirac equation (4.5) is. Nevertheless we will see that the supersymmetric graded decompositions can be used to derive the explicit form of the nilpotent charges in the non-supersymmetric case as well.

\section{Non-BPS solutions in five-dimensional theories}

The second class of solutions appear in the theories that can be obtained by toroidal compactification of (super)gravity theories in five dimensions to four dimensions. Inspection of the list of theories displayed in [6] shows that this is the case whenever $\mathfrak{H}^{*}$ is semi-simple. The scalar fields of the five-dimensional theory then parametrise a symmetric space $\mathfrak{G}_{5} / \mathfrak{H}_{5}$, and the isometry group $\mathfrak{G}_{5}$ acts on the abelian vector fields in a faithful representation $\mathfrak{l}_{5}$. One then has the following three-graded decomposition of $\mathfrak{g}_{4}$ and the associated four-graded decomposition of $\mathfrak{l}_{4}$,

$$
\mathfrak{g}_{4} \cong \mathfrak{l}_{5}^{(-2)} \oplus\left(\mathfrak{g l}_{1} \oplus \mathfrak{g}_{5}^{(0)}\right) \oplus \mathfrak{l}_{5}^{(2)}, \quad \mathfrak{l}_{4} \cong \mathbf{1}^{(-3)} \oplus \mathfrak{l}_{5}^{(-1)} \oplus \mathfrak{l}_{5}^{(1)} \oplus \mathbf{1}^{(3)}
$$

The maximal compact subalgeba $\mathfrak{h}_{4} \cong \mathfrak{h}_{5} \oplus \mathfrak{l}_{5}$ admits a non-compact real form $\mathfrak{h}_{4}^{*} \cong \mathfrak{h}_{5} \oplus i \mathfrak{l}_{5}$ which appears in the pseudo-Riemannian coset space $\mathfrak{G}_{4} / \mathfrak{H}_{4}^{*}$ of the Euclidean four-dimensional theory obtained by dimensional reduction along the timecoordinate. Using the graded decomposition one obtains the following result: because $\mathfrak{l}_{4}$ defines a complex irreducible representation of $\mathfrak{h}_{4} \subset \mathfrak{g}_{4}$, it decomposes into two conjugate real irreducible representations, $\mathfrak{l}_{4}^{*}$ and $\overline{\mathfrak{l}}_{4}^{*}$, of $\mathfrak{h}_{4}^{*} \subset \mathfrak{g}_{4}$. This property can be understood as follows. Let us define the four-dimensional field strength $\mathcal{F}$ in the $\mathbf{1} \oplus \mathfrak{l}_{5}$ representation, as well as $\mathcal{G} \equiv \delta \mathcal{L} / \delta \mathcal{F}$ (which in general depends on the scalar fields) such that the equations of motion of the abelian vector fields are $d \star \mathcal{G}=0$. The $\mathfrak{H}_{4}$ invariant form of the action on a four-dimensional pseudo-Riemannian space-time involves the complex combination $\star \mathcal{G}+i \mathcal{F}$ which transforms in the complex representation $\mathfrak{l}_{4}$ of $\mathfrak{H}_{4}$. This representation is complex because $\star^{2}=-1$ for a pseudo-Riemannian metric, whereas the $\mathfrak{H}_{4}^{*}$-invariant form of the action on a four-dimensional Riemannian space involves the two independent real combinations $\star \mathcal{G} \pm \mathcal{F}$ which transform in the real representation $\mathfrak{l}_{4}^{*}$ and its dual $\overline{\mathfrak{l}}_{4}^{*}$, respectively. As an example consider $\mathcal{N}=8$ supergravity, where $\star \mathcal{G}+i \mathcal{F}$ belongs to the complex representation 28 of $S U(8)$, whereas in the Euclidean signature theory the corresponding combinations $\star \mathcal{G} \pm \mathcal{F}$ belong to conjugate real representations $\mathbf{2 8}$ and $\overline{\mathbf{2 8}}$ of $S U^{*}(8)$.

It follows that when the four-dimensional theory descends from a five-dimensional one, the Lie algebra $\mathfrak{h}^{*} \cong \mathfrak{s l}_{2} \oplus \mathfrak{h}_{5} \oplus \mathbf{3} \otimes \mathfrak{l}_{5}$ admits the following three-graded decomposition

$$
\mathfrak{h}^{*} \cong \mathfrak{l}_{4}^{*(-2)} \oplus\left(\mathfrak{g l}_{1} \oplus \mathfrak{h}_{4}^{*}\right)^{(0)} \oplus \overline{\mathfrak{l}}_{4}^{*(2)}
$$


which gives an example of decomposition (3.17), with $\mathfrak{h}^{(4)} \cong \emptyset$ in this case. Indeed one obtains the five-graded decomposition of $\mathfrak{g} \ominus \mathfrak{h}^{*}$,

$$
\mathfrak{g} \ominus \mathfrak{h}^{*} \cong \mathbf{1}^{(-4)} \oplus \overline{\mathfrak{l}}_{4}^{*(-2)} \oplus\left(\mathfrak{g}_{4} \ominus \mathfrak{h}_{4}^{*}\right)^{(0)} \oplus \mathfrak{l}_{4}^{*(2)} \oplus \mathbf{1}^{(4)}
$$

such that $\mathfrak{l}_{4}^{*(2)} \oplus \mathbf{1}^{(4)}$ defines an abelian nilpotent subalgebra of $\mathfrak{g}$. One can thus define solutions of Papapetrou-Majumdar depending on $\operatorname{dim}\left[\mathfrak{l}_{5}\right]+2$ independent harmonic functions. As we will see, the most general asymptotically Minkowski solutions of this type are defined in function of charges lying in $\mathfrak{l}_{4}^{*(2)}$ and depend on $\operatorname{dim}\left[\mathfrak{l}_{5}\right]+1$ independent harmonic functions, and the extra-harmonic function corresponds to considering these extremal black holes in the background of pure NUT extremal black holes with vanishing horizon area.

\section{Maximal supergravity}

We now wish to illustrate the general discussion of the last sections through the example of the maximally supersymmetric supergravity. This theory being also the toroidal dimensional reduction of the maximally supersymmetric supergravity theory in five dimensions, it carries the two kinds of Papapetrou-Majumdar solutions discussed in the two preceding sections. They define the most general solutions of Papapetrou-Majumdar of the theory, which correspond to the only two $E_{8(8)}$ nilpotent orbits associated to the graded decomposition (3.16), respectively [8,21].

Let us first recall the form of the $E_{8(8)}$ Noether charge in the harmonic oscillator basis [8], with the mass and NUT charges $W=m+i n$, the electromagnetic central charges $Z_{i j}$ and the scalar charges $\Sigma_{i j k l}$. The latter are determined as functions of $W$ and $Z_{i j}$ through the characteristic equation (3.14). The state $|\mathscr{C}\rangle \in \mathfrak{e}_{8(8)} \ominus \mathfrak{s o}^{*}(16)$ is explicitly given by

$$
\begin{aligned}
|\mathscr{C}\rangle= & \left(W+Z_{i j} a^{i} a^{j}+\Sigma_{i j k l} a^{i} a^{j} a^{k} a^{l}+\frac{1}{6 !} \varepsilon_{i j k l m n p q} Z^{p q} a^{i} \cdots a^{n}\right. \\
& \left.+\frac{1}{8 !} \varepsilon_{i j k \operatorname{lmnpq}} \bar{W} a^{i} \cdots a^{q}\right)|0\rangle \\
= & (1+\star)\left(W+Z_{i j} a^{i} a^{j}+\frac{1}{2} \Sigma_{i j k l} a^{i} a^{j} a^{k} a^{l}\right)|0\rangle
\end{aligned}
$$

where $\star$ is the anti-involution defining the 128-dimensional chiral Majorana-Weyl representations of $\operatorname{Spin}^{*}$ (16) [8]. In the following subsections we will exhibit the form of $|\mathscr{C}\rangle$ explicitly for various kinds of extremal black holes.

\section{$6.1 \frac{1}{8}$ BPS solutions}

For a generic $\frac{1}{8}$ BPS solution preserving the supersymmetry generators associated to the Killing spinor parameters

$$
\epsilon_{\alpha}^{1}+\varepsilon_{\alpha \beta} \epsilon_{2}^{\beta}=0, \quad \epsilon_{\alpha}^{\bar{A}}=0
$$


with $\bar{A}$ running from 3 to 8 , the Noether charge $|\mathscr{C}\rangle$ must satisfy

$$
\left(a^{1}+a_{2}\right)|\mathscr{C}\rangle=\left(a^{2}-a_{1}\right)|\mathscr{C}\rangle=0
$$

The general solution can be written as

$$
\mathscr{C}=(1+\star)\left(1+a^{1} a^{2}\right)\left(W+Z_{\bar{A} \bar{B}} a^{\bar{A}} a^{\bar{B}}\right)|0\rangle
$$

This spinor evidently obeys the Majorana-Weyl condition $\star|\mathscr{C}\rangle=|\mathscr{C}\rangle$, and to see that it still satisfies (6.3) in accordance with (4.19) one uses the identity

$$
(1+\star)\left(1+a^{1} a^{2}\right)\left(W+Z_{\bar{A} \bar{B}} a^{\bar{A}} a^{\bar{B}}\right)|0\rangle=\left(1+a^{1} a^{2}\right)(1+\tilde{\star})\left(W+Z_{\bar{A} \bar{B}} a^{\bar{A}} a^{\bar{B}}\right)|0\rangle
$$

where $\tilde{\star}$ is the anti-involution for $\mathfrak{s o}^{*}(12)$. The grading generator $D_{\frac{1}{8}} \in \mathfrak{s o}^{*}(16)$ is

$$
D_{\frac{1}{8}} \equiv a^{1} a^{2}-a_{1} a_{2}
$$

and obeys

$$
D_{\frac{1}{8}}|\mathscr{C}\rangle=|\mathscr{C}\rangle
$$

for any value of $Z_{\bar{A} \bar{B}}$. It gives rise to the following five-graded decomposition of $\mathfrak{s o}^{*}(16)$

$$
\mathfrak{s o}^{*}(16) \cong \mathbf{1}^{(-2)} \oplus(\mathbf{2} \otimes \mathbf{1 2})_{\mathbb{R}}^{(-1)} \oplus\left(\mathfrak{g l}_{1} \oplus \mathfrak{s u}(2) \oplus \mathfrak{s o}^{*}(12)\right)^{(0)} \oplus(\mathbf{2} \otimes \mathbf{1 2})_{\mathbb{R}}^{(1)} \oplus \mathbf{1}^{(2)}
$$

The associated charge $\mathscr{C} \in \mathfrak{e}_{8(8)} \ominus \mathfrak{s o}^{*}(16)$ is left invariant by $\mathfrak{s u}(2)$ and transforms as a Majorana-Weyl spinor $\mathbf{3 2}$ of $\mathfrak{s o}^{*}(12)$, with the decomposition

$$
\mathfrak{e}_{8(8)} \ominus \mathfrak{s o}^{*}(16) \cong \mathbf{3 2}_{+}^{(-1)} \oplus\left(\mathbf{2} \otimes \mathbf{3 2}_{-}\right)_{\mathbb{R}}^{(0)} \oplus \mathbf{3 2}_{+}^{(1)}
$$

To see this explicitly, we invoke (6.5) once more.

The action of the grade zero component $\mathbb{R}_{+}^{*} \times S U(2) \times S p i n^{*}(12) \subset S p i n^{*}(16)$, preserves the given supersymmetry charges by construction. In a similar way as for the action of $\operatorname{Spin}^{*}(16)$ on the Kerr solutions, the moduli space of regular black holes preserving these four supersymmetry charges is the closure of the $\mathbb{R}_{+}^{*} \times \operatorname{Spin}^{*}(12)$ 
orbit of a given regular solution that can be chosen to correspond to the charge matrix $\mathscr{C}=\left(1+a^{1} a^{2}\right)(1+\tilde{\star})|0\rangle$. This orbit is 9

$$
\mathcal{M}_{1}^{1} \cong \frac{\mathbb{R}_{+}^{*} \times \operatorname{Spin}^{*}(12)}{S U(6)} \subset \mathcal{M}_{1} \cong \frac{\operatorname{Spin}^{*}(16)}{\operatorname{Ic}(S U(2) \times S U(6))}
$$

This embedding is a refinement of the stratification (4.3), with the following notation: by $\mathcal{M}_{n}^{m}$ for $m \leq n$ we generally designate the space of $\frac{n}{\mathcal{N}}$-BPS solutions which are such that $m$ out of $n$ super symmetry charges are shared between all of its elements (which implies $\mathcal{M}_{n}^{m} \subset \mathcal{M}_{n}^{m^{\prime}}$ for $m>m^{\prime}$ ). In other words, while $\mathcal{M}_{1}$ contains all $\frac{1}{8}$-BPS solutions, irrespective of which supersymmetry charges are left invariant, $\mathcal{M}_{1}^{1}$ consists of all solutions that are $\frac{1}{8}$ supersymmetric with respect to the same supersymmetry charge. Consequently, superposing solutions with charges $\mathscr{C}_{m}$ from the orbit $\mathcal{M}_{1}^{1}$ according to the basic ansatz (3.1) will result in a multi-black hole solution that still preserves $\frac{1}{8}$-supersymmetry. By contrast, taking different $\mathscr{C}_{m}$ from the larger orbit $\mathcal{M}_{1}$ in general will lead to a solution that is no longer supersymmetric because the $\frac{1}{8}$ supersymmetries of its black hole 'constituents' do not match.

Positivity of the energy entails that $|W|$ is strictly greater than any of the eigenvalues of $Z_{\bar{A} \bar{B}}$, and that the quartic $E_{7(7)}$ invariant associated to it is strictly positive. Note that to avoid naked singularities, one must require moreover $W \in \mathbb{C} \backslash \mathbb{R}_{-}$, and $W \in \mathbb{R}_{+}^{*}$ for asymptotically Minkowski solutions. The boundary of the orbit (6.10) decomposes into orbits corresponding to black holes with vanishing horizon area ${ }^{10}$

$$
\begin{aligned}
& \mathcal{M}_{1}^{1} \cong \quad \frac{\operatorname{Spin}^{*}(12)}{\operatorname{Sp}(3) \ltimes \mathbf{1 4}_{2}} \quad \subset \mathcal{M}_{1^{\circ}} \cong \frac{\operatorname{Spin}^{*}(16)}{\mathbb{R}_{+}^{*} \ltimes I C\left(\operatorname{SU}(2) \times \operatorname{Sp}(3) \ltimes \mathbf{1 4}_{2}\right)} \\
& \mathcal{M}_{2}^{1} \cong \frac{\operatorname{Spin}^{*}(12)}{(\operatorname{SU}(2) \times \operatorname{Spin}(1,6)) \ltimes\left((\mathbf{2} \otimes \mathbf{8})_{\mathbb{R}} \oplus \mathbf{1}\right)} \subset \mathcal{M}_{2} \cong \frac{\operatorname{Spin}^{*}(16)}{\left.\left(\operatorname{SU}^{*}(4) \times \operatorname{Spin}(1,6)\right) \ltimes(\mathbf{4} \otimes \mathbf{8})_{\mathbb{R}} \oplus \mathbf{6}\right)} \\
& \mathcal{M}_{4}^{1} \cong \quad \frac{\operatorname{Spin}^{*}(12)}{\operatorname{SU}(6) \ltimes \mathbf{1 5}} \quad \subset \mathcal{M}_{4} \cong \quad \frac{\operatorname{Spin}^{*}(16)}{S U^{*}(8) \ltimes \mathbf{2 8}}
\end{aligned}
$$

with the notation from [8] (see especially Eq. (5.17) there) for the embedding of the various 'BPS strata' into the stratified space of regular extremal solutions of the characteristic equation (3.14). So in particular, and in accordance with the notation introduced above, the 'BPS substrata' $\mathcal{M}_{2}^{1}$ and $\mathcal{M}_{4}^{1}$ correspond to $\frac{1}{4}$ and $\frac{1}{2}$ BPS black holes, all of which share the same $\frac{1}{8}$ supersymmetry, respectively (while by $\mathcal{M}_{1}$ 。 we denote the 9 See [8] for our notations (which are not standard): by ' $I c$ ' we designate the parabolic extension of
$S U(2) \times S U(6)$ by the grade-one translation generators and the grade-two central charge

$$
I c(S U(2) \times S U(6)) \equiv(S U(2) \times S U(6)) \ltimes\left((\mathbf{2} \otimes \mathbf{6})^{(1)} \oplus \mathbf{1}^{(2)}\right)
$$

Note also that the $S U$ (2) factors in the quotient defining $\mathcal{M}_{1}^{1}$ in (6.10) 'cancel out'.

10 where $I c\left(S U(2) \times S p(3) \ltimes \mathbf{1 4}_{2}\right)$ is the contracted form of $I c(S U(2) \times S U(6))$ :

$$
(S U(2) \times S p(3)) \ltimes\left(\left((\mathbf{2} \otimes \mathbf{6})_{+} \oplus \mathbf{1 4}_{2}\right)^{(1)} \oplus(\mathbf{2} \otimes \mathbf{6})_{+}^{(2)} \oplus \mathbf{1}^{(3)}\right)
$$


stratum of general $\frac{1}{8}$ BPS solutions of vanishing horizon area). In all three cases the $E_{7(7)}$ invariant $\diamond\left(W^{-\frac{1}{2}} Z\right)$ associated to $\mathscr{C}$ vanishes, and zero, one or three of the eigenvalues of $Z_{\bar{A} \bar{B}}$ are equal to $W$ in modulus, respectively. These orbits correspond to the various Majorana-Weyl spinors of null type which turn out to be slightly more complicated than in the case of the null vectors of $S O(p, q)$. For example, the $\operatorname{Spin}^{*}(12)$ Majorana-Weyl spinors associated to $\frac{1}{2}$ BPS solutions are pure in the sense of Cartan.

By virtue of the grading, any linear combination of these charges satisfies $\mathscr{C}^{5}=0$, and moreover, the commutator of any two of them gives

$$
\begin{aligned}
& {\left[\mathscr{C}_{n}, \mathscr{C}_{m}\right]=\left(W_{n} \bar{W}_{m}-\bar{W}_{n} W_{m}-2 Z_{n \bar{A} \bar{B}} Z_{m}^{\bar{A} \bar{B}}+2 Z_{n}^{\bar{A} \bar{B}} Z_{m \bar{A} \bar{B}}\right)} \\
& \times\left(a^{1}+a_{2}\right)\left(a^{2}-a_{1}\right) \in \mathbf{1}^{(2)}
\end{aligned}
$$

The generator $\left(a^{1}+a_{2}\right)\left(a^{2}-a_{1}\right)$ of $\mathfrak{s o}^{*}(16)$ leaves invariant the Killing spinor associated to the charges $\mathscr{C}_{n}$, and the Killing spinor equation trivially reduces to

$$
d \epsilon_{\alpha}^{A}=0
$$

We have thus derived the most general $\frac{1}{8}$ BPS solution of maximal supergravity, which depends on 32 independent harmonic functions associated to the independent components of the $\operatorname{Spin}^{*}(12)$ Majorana-Weyl spinor. Of course in general, some of the $\mathscr{C}_{n}$ can preserve more supersymmetry, in such a way that each black hole can be either a generic $\frac{1}{8}$ BPS black hole carrying one of the 32 linearly independent charges of $\mathcal{M}_{1}^{1}$, or a $\frac{1}{8}$ BPS black hole of vanishing horizon area carrying one of the 31 linearly independent charges of $\mathcal{M}_{1}^{1}$, or a $\frac{1}{4}$ BPS black hole carrying one of the 25 linearly independent charges of $\mathcal{M}_{2}^{1}$, or a $\frac{1}{2}$ BPS black hole carrying one of the 16 linearly independent charges of $\mathcal{M}_{4}^{1}$. All these solutions can be understood as $\frac{1}{2}$ BPS multiblack holes of the magic $\mathcal{N}=2$ supergravity associated to the quaternions [22]. Within the latter truncation, $D_{\frac{1}{8}}$ is a non compact generator of the $\mathfrak{s l}_{2}$ component of $\mathfrak{s l}_{2} \oplus \mathfrak{s o}^{*}(12) \subset \mathfrak{e}_{7(-5)}$, which decomposes $\mathfrak{e}_{7(-5)}$ as

$$
\mathfrak{e}_{7(-5)} \cong \mathbf{1}^{(-2)} \oplus \mathbf{3 2}_{+}^{(-1)} \oplus\left(\mathfrak{g l}_{1} \oplus \mathfrak{s o}^{*}(12)\right)^{(0)} \oplus \mathbf{3 2}_{+}^{(1)} \oplus \mathbf{1}^{(2)}
$$

exhibiting the fact that these solutions can be understood inside the $\mathcal{N}=2$ truncated theory.

\section{$6.2 \frac{1}{4}$ BPS solutions}

For solutions preserving $\frac{1}{4}$ supersymmetry the charge state is of the form (using (4.19) once again)

$$
|\mathscr{C}\rangle=e^{\frac{1}{2} \Omega_{A B} a^{A} a^{B}}\left(W+Z_{\bar{A} \bar{B}} a^{\bar{A}} a^{\bar{B}}+\frac{1}{24} \varepsilon_{\bar{A} \bar{B} \bar{C} \bar{D}} \bar{W} a^{\bar{A}} a^{\bar{B}} a^{\bar{C}} a^{\bar{D}}\right)|0\rangle
$$


in an appropriate basis, where $\Omega_{A B}$ is a complex-selfdual symplectic form of $\mathbb{C}^{4}$ and $Z_{\bar{A} \bar{B}}$ is complex-selfdual with respect with the complementary $U(4)$. The expression in parentheses is thus a chiral spinor of $\operatorname{Spin}^{*}(8)$; by triality and because of the isomorphism $\operatorname{Spin}^{*}(8) \cong \operatorname{Spin}(2,6)$ it can be equivalently viewed as a vector of $S O(2,6)$. The Killing spinors for (6.15) then satisfy

$$
\epsilon_{\alpha}^{A}+\varepsilon_{\alpha \beta} \Omega^{A B} \epsilon_{B}^{\beta}=0, \quad \epsilon_{\alpha}^{\bar{A}}=0
$$

The associated grading operator of $\mathfrak{s o}^{*}(16)$ is

$$
D_{\frac{1}{4}} \equiv \frac{1}{2}\left(\Omega_{A B} a^{A} a^{B}-\Omega^{A B} a_{A} a_{B}\right)
$$

and we now have

$$
D_{\frac{1}{4}}|\mathscr{C}\rangle=2|\mathscr{C}\rangle
$$

The corresponding five-graded decomposition of $\mathfrak{s o}^{*}(16)$ is

$$
\mathfrak{s o}^{*}(16) \cong \mathbf{6}^{(-2)} \oplus(\mathbf{4} \otimes \mathbf{8})_{\mathbb{R}}^{(-1)} \oplus\left(\mathfrak{g l}_{1} \oplus \mathfrak{s u}^{*}(4) \oplus \mathfrak{s o}^{*}(8)\right)^{(0)} \oplus(\mathbf{4} \otimes \mathbf{8})_{\mathbb{R}}^{(1)} \oplus \mathbf{6}^{(2)}
$$

One computes that $|\mathscr{C}\rangle$ transforms as a Majorana-Weyl $\operatorname{Spin}^{*}(8)$ spinor with respect with $S U^{*}(4) \times \operatorname{Spin}^{*}(8) \cong \operatorname{Spin}(5,1) \times \operatorname{Spin}(2,6)$, i.e. as a vector of $\operatorname{SO}(2,6)$. Indeed

$$
\mathfrak{e}_{8(8)} \ominus \mathfrak{s o}^{*}(16) \cong \mathbf{8}_{+}^{(-2)} \oplus\left(\mathbf{4} \otimes \mathbf{8}_{-}\right)_{\mathbb{R}}^{(-1)} \oplus\left(\mathbf{6} \otimes \mathbf{8}_{+}\right)^{(0)} \oplus\left(\mathbf{4} \otimes \mathbf{8}_{-}\right)_{\mathbb{R}}^{(1)} \oplus \mathbf{8}_{+}^{(2)}
$$

where by $\mathbf{8}$ and $\mathbf{8}_{ \pm}$we denote the three inequivalent fundamental representations of $\operatorname{Spin}^{*}(8)$. In accordance with the grading (6.20) a charge matrix of the form (6.15) transforms as $\mathbf{8}_{+}$and defines a nilpotent abelian subalgebra $\mathbb{R}^{2+6}$ of $\mathfrak{e}_{8(8)}$. The corresponding moduli space is

$$
\mathcal{M}_{2}^{2} \cong \frac{\mathbb{R}_{+}^{*} \times S O(2,6)}{S O(1,6)} \subset \mathcal{M}_{2} \cong \frac{\operatorname{Spin}^{*}(16)}{\left(S U^{*}(4) \times \operatorname{Spin}(1,6)\right) \ltimes\left((\mathbf{4} \times \mathbf{8})_{\mathbb{R}} \oplus \mathbf{6}\right)}
$$

The subgroup $S O(1,6)$ here is the isotropy group of a timelike vector of $S O(2,6)$. In order for the corresponding multi-black holes solutions to be regular, one must thus require the charge matrices in (6.21) to correspond to non-space-like vectors. When the norm of the $S O(2,6)$ vector goes to zero, we reach the boundary of $\mathcal{M}_{2}^{2}$ corresponding to the subspace of $\frac{1}{2}$ BPS charge matrices belonging to

$$
\mathcal{M}_{4}^{2} \cong \frac{S O(2,6)}{I S O(1,5)} \subset \mathcal{M}_{4} \cong \frac{\operatorname{Spin}^{*}(16)}{S U^{*}(8) \ltimes \mathbf{2 8}}
$$


where we again employ the notation in (6.10). So we conclude that the general $\frac{1}{4}$ BPS solutions depend on eight independent harmonic functions associated to the linearly independent non-spacelike $S O(2,6)$ vectors. Note nevertheless that even if all the vectors are chosen to be null, the solution only preserves one quarter supersymmetry since, although each black hole is $\frac{1}{2}$ BPS, there are only eight supercharges that are preserved by each one of them. As the most general $\frac{1}{8}$ BPS solutions were possibly understood within the magic $\mathcal{N}=2$ truncated theory associated to the quaternions, the general $\frac{1}{4}$ BPS solutions can be understood within $\mathcal{N}=4$ supergravity coupled to six vector multiplets. In the latter truncation, $D_{\frac{1}{4}}$ is the generator of the $\mathfrak{s o}^{*}(8)$ R-symmetry group of the theory,

$$
\mathfrak{s o}^{*}(8) \cong \mathbf{6}^{(-2)} \oplus\left(\mathfrak{g l}_{1} \oplus \mathfrak{s u}^{*}(4)\right)^{(0)} \oplus \mathbf{6}^{(2)}
$$

such that

$$
\mathfrak{s o}(8,8) \ominus\left(\mathfrak{s o}^{*}(8) \oplus \mathfrak{s o}^{*}(8)\right) \cong \mathbf{8}_{+}^{(-2)} \oplus\left(\mathbf{6} \otimes \mathbf{8}_{+}\right)^{(0)} \oplus \mathbf{8}_{+}^{(2)}
$$

\section{$6.3 \frac{1}{2}$ BPS solutions}

For $\frac{1}{2}$ BPS solutions, the set of 16 supersymmetry charges is associated to the Killing spinors

$$
\epsilon_{\alpha}^{i}+\varepsilon_{\alpha \beta} \Omega^{i j} \epsilon_{j}^{\beta}=0
$$

where $\Omega_{i j}$ is a (generally complex) symplectic form of $\mathbb{C}^{8}$ satisfying $\Omega_{i k} \Omega^{j k}=\delta_{i}^{j}$ (with our usual convention $\Omega^{i j} \equiv\left(\Omega_{i j}\right)^{*}$ ). In this case the four-dimensional R-symmetry group $S U(8)$ permits to rotate $\Omega_{i j}$ to a diagonal basis

$$
\Omega_{i j} \hat{=} e^{i \omega}\left(\begin{array}{cc}
0 & \mathbb{1} \\
-\mathbb{1} & 0
\end{array}\right)
$$

but one cannot eliminate the overall phase $\omega$ in general. Equation (4.5) uniquely determines the charge matrix as

$$
|\mathscr{C}\rangle=m e^{-2 i \omega} e^{\frac{1}{2} \Omega_{i j} a^{i} a^{j}}|0\rangle
$$

where the overall phase factor $e^{-2 i \omega}$ ensures that $|\mathscr{C}\rangle$ is a Majorana spinor for any choice of $\omega$ via the identity

$$
e^{-2 i \omega} \Omega_{[i j} \Omega_{k l]}=e^{2 i \omega} \frac{1}{24} \varepsilon_{i j k l m n p q} \Omega^{m n} \Omega^{p q}
$$

Asymptotically Minkowskian $\frac{1}{2}$ BPS black holes thus correspond to $\omega=0$. The asymptotic central charges are then purely electric modulo an $S U(8)$ rotation. 
The general $\frac{1}{2}$ BPS solution is determined by one single harmonic function and the given $\frac{1}{2}$ BPS charge matrix. The relevant grading operator is

$$
D_{\frac{1}{2}} \equiv \frac{1}{2}\left(\Omega_{i j} a^{i} a^{j}-\Omega^{i j} a_{i} a_{j}\right)
$$

and satisfies $D_{\frac{1}{2}}|\mathscr{C}\rangle=4|\mathscr{C}\rangle$. It commutes with the generators of $\mathfrak{s u}^{*}(8)$ and its action on the nilpotent generators follows from

$$
\left[D_{\frac{1}{2}}, a^{i} \pm \Omega^{i j} a_{j}\right]= \pm\left(a^{i} \pm \Omega^{i j} a_{j}\right)
$$

and thus gives rise to the decomposition of $\mathfrak{s o}^{*}(16)$

$$
\mathfrak{s o}^{*}(16) \cong \mathbf{2 8}^{(-2)} \oplus\left(\mathfrak{g l}_{1} \oplus \mathfrak{s u}^{*}(8)\right)^{(0)} \oplus \overline{\mathbf{2 8}}^{(2)}
$$

such that

$$
\mathfrak{e}_{8(8)} \ominus \mathfrak{s o}^{*}(16) \cong \mathbf{1}^{(-4)} \oplus \overline{\mathbf{2 8}}^{(-2)} \oplus \mathbf{7 0}^{(0)} \oplus \mathbf{2 8}^{(2)} \oplus \mathbf{1}^{(4)}
$$

and $|\mathscr{C}\rangle \equiv\left|\mathscr{C}^{(4)}\right\rangle \in \mathbf{1}^{(4)}$. Let us point out once again that the level \pm 2 representations of $S U^{*}(8)$ are both real, but dual to one another, and correspond to independent real combinations of the field strengths, as explained in Sect. 5.

\subsection{Non-BPS solutions}

The results of the preceding section can be summarised by characterising the relevant vector space of $\mathfrak{g} \ominus \mathfrak{h}^{*}$, i.e. $\mathfrak{g}^{(2)} \ominus \mathfrak{h}^{(2)} \cong \mathbf{3 2}, \mathbf{8}$ and $\mathbf{1}$, for the $\frac{1}{8}$ BPS, the $\frac{1}{4}$ BPS and the $\frac{1}{2}$ BPS solutions, respectively. One can now construct non-BPS multi-particle solutions in a similar way. Namely, the charge matrices of non-BPS extremal solutions are associated to the same graded decomposition (6.32) of $\mathfrak{e}_{8(8)}$ as the maximally supersymmetric ones. The only difference is that the associated charge matrices now belong to the grade one component of (6.32), which defines an abelian nilpotent subalgebra $\mathbb{R}^{1+27} \subset \mathfrak{e}_{8(8)}$. The relevant ( $\omega$-dependent) generator $\mathbf{H} \in \mathfrak{s o}^{*}(16)$ is thus identical with $D_{\frac{1}{2}}$, cf. (6.29).

The simplest solution of this type is obtained from the state

$$
\left|\mathscr{C}^{(2)}\right\rangle=(1+\star)\left(1+\frac{1}{4} \Omega_{i j} a^{i} a^{j}\right) i e^{-2 i \omega}|0\rangle
$$

where the overall phase is determined such that the four-form component of

$$
\left.(\mathbf{H}-2)\left|\mathscr{C}^{(2)}\right\rangle\right|_{4 \text {-form }}=\frac{1}{8}(1+\star) i e^{-2 i \omega} \Omega_{i j} \Omega_{k l} a^{i} a^{j} a^{k} a^{l}|0\rangle=0
$$


vanishes due to the action of the projection operator $\frac{1}{2}(1+\star)$. In order to obtain nonBPS asymptotically Minkowskian extremal black holes, one must therefore choose $\omega=\frac{\pi}{4}$. The top grade component then becomes associated to a pure $\frac{1}{2}$ BPS Taub-NUT black hole as would be obtained from the level four charge matrix

$$
\left|\mathscr{C}^{(4)}\right\rangle=\operatorname{in} e^{\frac{1}{2} \Omega_{i j} a^{i} a^{j}}|0\rangle
$$

The charge matrix of grade two depends on the associated mass $m$ and a rank two tensor $Q_{i j}$ satisfying ${ }^{11}$

$$
Q_{i j}=\Omega_{i k} \Omega_{j l} Q^{k l}, \quad \Omega^{i j} Q_{i j}=0
$$

such that

$$
\left|\mathscr{C}^{(2)}\right\rangle=(1+\star)\left(1+\frac{1}{4} \Omega_{i j} a^{i} a^{j}\right)\left(m+Q_{i j} a^{i} a^{j}\right)|0\rangle
$$

In this way one obtains multi-particle solutions depending on 28 harmonic functions. Although the symplectic form is not an invariant tensor of $S U^{*}(8)$, the reality condition

$$
Z_{i j}=\Omega_{i k} \Omega_{j l} Z^{k l}
$$

is nonetheless preserved by $S U^{*}(8)$, and it defines the real 28 representation of $S U^{*}(8)$. This is also the representation to which belong the asymptotic central charges

$$
Z_{i j}=\frac{m}{4} \Omega_{i j}+Q_{i j}
$$

In contradistinction to the $\frac{1}{2}$ BPS asymptotically Minkowski black holes, the non-BPS ones are therefore purely dyonic $\left(\omega=\frac{\pi}{4}\right)$, up to an $S U(8)$ rotation.

$\left|\mathscr{C}^{(2)}\right\rangle$ corresponds to a regular black holes as long as the eigenvalues of $Z_{i j}$ are less than or equal to $m$ in modulus. The $E_{7(7)}$ quartic invariant $\nabla(Z)$ is then negative,

$$
\begin{aligned}
\diamond(Z)= & -\frac{m^{4}}{16}+\frac{m^{2}}{4} Q^{i j} Q_{i j}+\frac{m}{6} \Omega_{i j} Q^{j k} Q_{k l} Q^{l i}-\frac{1}{2}\left(Q^{i j} Q_{i j}\right)^{2} \\
& +2 Q_{i j} Q^{j k} Q_{k l} Q^{l i} \leq 0
\end{aligned}
$$

In order to spell out the these conditions explicitly, it is useful to find a basis in which both $\Omega_{i j}$ and $Q_{i j}$ are block diagonal (which always exists thanks to the reality condition (6.36))

\footnotetext{
11 Which means that $Q_{i j}$ lies in the 27 representation of $S p(4) \subset S U(8)$ which leaves invariant $\Omega_{i j}$.
} 


$$
\Omega_{i j} \hat{=} e^{\frac{i \pi}{4}}\left(\begin{array}{cc}
0 & 1 \\
-1 & 0
\end{array}\right) \otimes \mathbb{1}, \quad Q_{i j} \hat{=} \frac{e^{\frac{i \pi}{4}}}{2}\left(\begin{array}{cc}
0 & 1 \\
-1 & 0
\end{array}\right) \otimes\left(\begin{array}{cccc}
\rho_{1} & 0 & 0 & 0 \\
0 & \rho_{2} & 0 & 0 \\
0 & 0 & \rho_{3} & 0 \\
0 & 0 & 0 & -\rho_{1}-\rho_{2}-\rho_{3}
\end{array}\right)
$$

The three real parameters $\rho_{i}$ are constrained to lie in the tetrahedron defined by the conditions $\rho_{i} \leq \frac{m}{2}$ for $i=1,2,3$ and $-\frac{m}{2} \leq \rho_{1}+\rho_{2}+\rho_{3}$. In this basis, the $E_{7(7)}$ invariant reads

$$
\diamond(Z)=-\left(\frac{m}{2}-\rho_{1}\right)\left(\frac{m}{2}-\rho_{2}\right)\left(\frac{m}{2}-\rho_{3}\right)\left(\frac{m}{2}+\rho_{1}+\rho_{2}+\rho_{3}\right)
$$

and one checks easily that it is negative inside the tetrahedron and that it vanishes on its faces. In the absence of NUT charge, one expects the horizon area of such black holes to be given by

$$
A_{\mathscr{H}}=4 \pi \sqrt{-\diamond(Z)}
$$

Such multi-black hole solutions include non-BPS black holes corresponding to one of the 28 linearly independent charge matrices in

$$
\frac{\mathrm{R}_{+}^{*} \times S U^{*}(8)}{S p(4)} \subset \frac{\operatorname{Spin}^{*}(16)}{S p(4) \ltimes \mathbf{2 7}}
$$

(for which the parameters $\rho_{i}$ lie inside the tetrahedron) as well as $\frac{1}{8}$ BPS black holes corresponding to one of the 27 linearly independent charge matrices in

$$
\frac{S U^{*}(8)}{(S U(2) \times S p(3)) \ltimes(\mathbf{2} \otimes \mathbf{6})_{\mathbb{R}}} \subset \frac{\operatorname{Spin}^{*}(16)}{\mathrm{R}_{+}^{*} \ltimes I c\left(S U(2) \times S p(3) \ltimes \mathbf{1 4}_{2}\right)}
$$

(for which $\rho_{i}$ lie on a face of the tetrahedron). Finally, $\frac{1}{4}$ BPS black holes correspond to one of the 22 linearly independent charge matrix in

$$
\frac{S U^{*}(8)}{\left(S U^{*}(4) \times S p(2)\right) \ltimes(\mathbf{4} \otimes \mathbf{4})_{\mathbb{R}}} \subset \frac{\operatorname{Spin}^{*}(16)}{(\operatorname{Spin}(5,1) \times \operatorname{Spin}(1,6)) \ltimes\left((\mathbf{4} \otimes \mathbf{8})_{\mathbb{R}} \oplus \mathbf{6}\right)}
$$

(with $\rho_{i}$ lying on an edge of the tetrahedron) and $\frac{1}{2}$ BPS black holes to one of the $13+1$ linearly independent charge matrices in

$$
\frac{S U^{*}(8)}{\left(S U^{*}(6) \times S p(1)\right) \ltimes(\mathbf{6} \otimes \mathbf{2})_{\mathbb{R}}} \cup \mathbb{R} \subset \frac{\operatorname{Spin}^{*}(16)}{S U^{*}(8) \ltimes \mathbf{2 8}}
$$


(corresponding to $\rho_{i}$ lying on a vertex of the tetrahedron). This set includes the charge matrix (6.35) lying in the component of grade four $\mathbf{1}^{(4)}$.

Note that these solutions are completely different from the BPS ones. To see this, we note that the decomposition of $\mathfrak{e}_{8(8)}$ associated to a $\frac{1}{2}$ BPS charge matrix within the graded decomposition associated to either the $\frac{1}{8}$ BPS solutions or the non-BPS extremal solutions lead to equivalent decomposition in terms of representations of $S U(2) \times S U^{*}(6)$. The charge matrix in the neighbourhood of this charge which define $\frac{1}{2}$ BPS black holes which do not interact with the $\frac{1}{2}$ BPS black hole associated to the latter, can be obtained by acting with either $\mathfrak{s o}^{*}(12)$ or $\mathfrak{s u}^{*}(8)$, for $\frac{1}{8}$ BPS and non-BPS solutions, respectively. The action of $\mathfrak{s o}^{*}(12)$ generates new components in the $\mathbf{1 5}$ of $S U^{*}(6)$ whereas the action of $\mathfrak{s u}(8)$ generates new components in the $(\mathbf{2} \otimes \mathbf{6})_{\mathbb{R}}$ of $S U(2) \times S U^{*}(6)$, which shows that they are inequivalent.

Acknowledgments We are grateful to Boris Pioline and Kelly Stelle for discussions and comments.

Open Access This article is distributed under the terms of the Creative Commons Attribution Noncommercial License which permits any noncommercial use, distribution, and reproduction in any medium, provided the original author(s) and source are credited.

\section{References}

1. Papapetrou, A.: A static solution of the equations of the gravitational field for an arbitrary charge distribution. Proc. R. Ir. Acad. A 51, 191 (1945)

2. Majumdar, S.D.: A class of exact solutions of Einstein's field equations. Phys. Rec. 72, 390 (1945)

3. Bates, B., Denef, F.: Exact solutions for supersymmetric stationary black hole composites [hep-th/ 0304094]

4. Ferrara, S., Kallosh, R., Strominger, A.: $\mathcal{N}=2$ extremal black holes. Phys. Rev. D 52, 5412 (1995) [hep-th/9508072]

5. Ferrara, S., R. Kallosh.: Supersymmetry and attractors. Phys. Rev. D 54, 1514 (1996) [hep-th/9602136]

6. Breitenlohner, P., Maison, D., Gibbons, G.W.: Four-dimensional black holes from Kaluza-Klein theories. Commun. Math. Phys. 120, 295 (1988)

7. Breitenlohner, P., Maison, D.: On nonlinear sigma-models arising in (super-)gravity. Commun. Math. Phys. 209, 785 (2000) [gr-qc/9806002]

8. Bossard, G., Nicolai, H., Stelle, K.S.: Universal BPS structure of stationary supergravity solutions, [hep-th/0902.4438]

9. Clement, G., Galtsov, D.V.: Stationary BPS solutions to dilaton-axion gravity. Phys. Rev. D 54, 6136 (1996) [hep-th/9607043]

10. Gunaydin, M., Neitzke, A., Pioline, B., Waldron, A.: BPS black holes, quantum attractor flows and automorphic forms. Phys. Rev. D 73, 084019 (2006) [hep-th/0512296]

11. Gaiotto, D., Li, W.W., Padi, M.: Non-supersymmetric attractor flow in symmetric spaces. JHEP 0712, 093 (2007) [hep-th/0710.1638]

12. Cremmer, E., Julia, B.: The $S O(8)$ supergravity. Nucl. Phys. B 159, 141 (1979)

13. Bossard, G.: The extremal black holes of $\mathcal{N}=4$ supergravity from $\mathfrak{s o}(8,2+n)$ nilpotent orbits Gen. Relativ. Gravit. (in press) [hep-th/0906.1988]

14. Bellucci, S., Ferrara, S., Gunaydin, M., Marrani, A.: SAM lectures on extremal black holes in $d=4$ extended supergravity, [hep-th/0905.3739]

15. Hotta, K., Kubota, T.: Exact solutions and the attractor mechanism in non-BPS black holes. Prog. Theor. Phys. 118, 969 (2007) [hep-th/0707.4554]

16. Gimon, E.G., Larsen, F., Simon, J.: Black holes in supergravity: the non-BPS branch. JHEP 0801, 040 (2008) [hep-th/0710.4967]

17. Breitenlohner, P., Maison, D.: Solitons in Kaluza-Klein theories. In: Morris, H., Dodd, R. (eds.) Solitons in General Relativity (1986) 
18. Stephani, H., Kramer, D., MacCallum, M.A., Hoenselars, C., Herlt, E.: Exact Solutions of Einstein's Field Equations. Cambridge University Press, Cambridge (2003)

19. Collingwood, D.H., McGovern, W.M.: Nilpotent Orbits in Semisimple Lie Algebra. Van Nostrand Reinhold Mathematics Series, New York (1993)

20. Günaydin, M., Sierra, G., Townsend, P.K.: Exceptional supergravity theories and the magic square. Phys. Lett. B 133, 72 (1983)

21. Đoković, D.Ž.: The closure diagram for nilpotent orbits of the split real form of $E_{8}$. CEJM 4, 573 (2003)

22. Ferrara, S., Gimon, E.G., Kallosh, R.: Magic supergravities, $\mathcal{N}=8$ and black hole composites. Phys. Rev. D 74, 125018 (2006) [hep-th/0606211] 